\title{
Insular Cortex Projections to Nucleus Accumbens Core Mediate Social Approach to Stressed Juvenile Rats
}

\author{
Morgan M. Rogers-Carter, Anthony Djerdjaj, K. Bates Gribbons, Juan A. Varela, and $₫$ John P. Christianson \\ Psychology Department, Boston College, Chestnut Hill, Massachusetts 02467
}

\begin{abstract}
Social interactions are shaped by features of the interactants, including age, emotion, sex, and familiarity. Age-specific responses to social affect are evident when an adult male rat is presented with a pair of unfamiliar male conspecifics, one of which is stressed via two foot shocks and the other naive to treatment. Adult test rats prefer to interact with stressed juvenile (postnatal day 30, PN30) conspecifics but avoid stressed adult (PN50) conspecifics. This pattern depends upon the insular cortex (IC), which is anatomically connected to the nucleus accumbens core (NAc). The goal of this work was to test the necessity of IC projections to NAc during social affective behavior. Here, bilateral pharmacological inhibition of the NAc with tetrodotoxin ( $1 \mu \mathrm{M} ; 0.5 \mu \mathrm{l} /$ side) abolished the preference for stressed PN30, but did not alter interactions with PN50 conspecifics. Using a combination of retrograding tracing and c-Fos immunohistochemistry, we report that social interactions with stressed PN30 conspecifics elicit greater Fos immunoreactivity in IC $\rightarrow$ NAc neurons than interactions with naive PN30 conspecifics. Chemogenetic stimulation of IC terminals in the NAc increased social exploration with juvenile, but not adult, conspecifics, whereas chemogenetic inhibition of this tract blocked the preference to investigate stressed PN30 conspecifics, which expands upon our previous finding that optogenetic inhibition of IC projection neurons mediated approach and avoidance. These new findings suggest that outputs of IC to the NAc modulate social approach, which provides new insight to the neural circuitry underlying social decision-making.
\end{abstract}

Key words: chemogenetics; insular cortex; nucleus accumbens; rat; social behavior; social decision-making

Significance Statement

Social decision-making underlies an animal's behavioral response to others in a range of social contexts. Previous findings indicate the insular cortex (IC) and the nucleus accumbens (NAc) play important roles in social behaviors, and human neuroimaging implicates both IC and NAc in autism and other psychiatric disorders characterized by aberrant social cognition. To test whether IC projections to the NAc are involved in social decision-making, circuit-specific chemogenetic manipulations demonstrated that the IC $\rightarrow$ NAc pathway mediates social approach toward distressed juvenile, but not adult, conspecifics. This finding is the first to implicate this circuit in rodent socioemotional behaviors and may be a neuroanatomical substrate for integration of emotion with social reward.

\section{Introduction}

Social decision-making enables animals to respond to their social environments with flexible and context-appropriate behaviors

\footnotetext{
Received Feb. 7, 2019; revised Aug. 30, 2019; accepted Sept. 18, 2019

Author contributions: M.M.R.-C., K.B.G., J.A.V., and J.P.C. designed research; M.M.R.-C., A.D., K.B.G., J.A.V., and J.P.C. performed research; M.M.R.-C., A.D., K.B.G., J.A.V., and J.P.C. analyzed data; M.M.R.-C. and J.P.C. wrote the first draft of the paper; M.M.R.-C., A.D., and J.P.C. edited the paper.

This work was supported by the National Science Foundation Graduate Research Fellowship Program (M.M.R.C.), the Gianinno Family (J.P.C.), and the National Institutes of Health (Grant MH109545 to J.P.C.). We thank Nancy McGilloway and the Boston College Animal Care Facility staff for the husbandry of research animals involved in this study. pAAV-hSyn-hM3D(Gq)-mCherry and pAAV-hSyn-hM4D(Gi)-mCherry were gifts from Bryan Roth (Addgene plasmids \#50474 and \#50475). pAAV-hSyn-mCherry was a gift from Karl Deisseroth (Addgene plasmid \#114472). The authors declare no competing financial interests.

Correspondence should be addressed to Morgan M. Rogers-Carter at rogersm13@ccf.org or John P. Christianson at j.christianson@bc.edu.

https://doi.org/10.1523/JNEUROSCI.0316-19.2019

Copyright $\odot 2019$ the authors
}

(Insel and Fernald, 2004; O'Connell and Hofmann, 2011). An important feature of social decision-making is that animals appraise the affective state of others to generate behavioral responses. Accordingly, a number of studies report that rodents demonstrate either prosocial or avoidance behaviors toward distressed others (reviewed by (Meyza et al., 2017). These responses are modulated by situational factors, including familiarity, age, social rank, sex and prior experience (Langford et al., 2006; Guzmán et al., 2009; Ishii et al., 2012; Kiyokawa et al., 2014; Li et al., 2014; Burkett et al., 2016; Meyza et al., 2017; Rogers-Carter et al., 2018a,b), which suggest that socioemotional information is integrated with context and situational factors to inform behavioral actions. However, how areas in the brain that integrate affective cues, external stimuli and physiology can inform behavioral responses remains elusive. 
Mechanistic studies have identified several structures in the social decision-making network (O'Connell and Hofmann, 2012) that produce flexible behavioral responses during social interactions (Hong et al., 2014; Lee et al., 2014; Felix-Ortiz et al., 2016; Yang and Tsai, 2017; Yao et al., 2017). The insular cortex (IC) is a site of multisensory integration (Rodgers et al., 2008; Gogolla et al., 2014; Gogolla, 2017) and it is highly interconnected with the social decision-making network positioning it as a hub for socioemotional information to be incorporated in social decision-making processes (Rogers-Carter and Christianson, 2019). In addition to detecting social cues, social decisionmaking uses reward valuation to determine behavioral responses, and socioemotional information is likely one factor that influences reward valuation and behavior during healthy and disordered social cognition (Kohls et al., 2012). The nucleus accumbens core (NAc) is implicated in reward (Wise, 2002) and IC provides a major cortical input to the NAc (Wright and Groenewegen, 1996). Thus, there is compelling evidence to investigate the IC $\rightarrow$ NAc projection as a possible pathway by which socially relevant sensory information reaches the reward system to inform social decisions.

We have reported a role for insular cortex (IC) in a social affective preference (SAP) test in which rats demonstrate agespecific responses to stressed others (Rogers-Carter et al., 2018b). Specifically, in the SAP test, experimental rats exhibit approach toward stressed juvenile rats, but avoid exploring stressed adult rats. Optogenetic silencing of IC pyramidal neurons abolished this pattern. Although the role of NAc in control of behavior during interactions with stressed individuals is unknown, it is well established that the NAc is critical to a number of social behaviors. NAc mediates social recognition (Ploeger et al., 1991) and reward with juvenile (Trezza et al., 2011) and adult conspecifics (Dölen et al., 2013). Regarding emotional cues, NAc encodes ultrasonic vocalization calls that convey stress (Willuhn et al., 2014) and shows enhanced dopaminergic transmission in subjects that observe a conspecific receiving foot shock (Wu et al., 1999), which suggests the NAc encodes the socioemotional cues present during interactions with stressed others.

In the SAP paradigm, we hypothesize that IC is integral to the binding of social and situational factors to inform social decisions which, via glutamatergic efferents to nodes in the social decisionmaking-network, can shape the pattern of circuit activity to favor approach or avoidant behavioral strategies. No prior mechanistic studies have explored the role of IC projection tracts in social behavior. Here, we used pharmacological and tract-specific manipulations to test if the IC $\rightarrow$ NAc projections are necessary for SAP behavior. We report that this tract is necessary and sufficient for approach toward stressed juvenile conspecifics, but not avoidance of stressed adult conspecifics.

\section{Materials and Methods}

Overview of approach. To determine the role of the IC $\rightarrow$ NAc tract in social affective behavior, we performed a series of experiments. First, to test the role of the NAc we used reversible inactivation by infusing tetrodotoxin (TTX) directly to NAc before SAP tests with either postnatal day 30 (PN30) juvenile or PN50 adult conspecifics. Next, we used retrograde tracing and Fos immunohistochemistry to quantify activity in IC $\rightarrow$ NAc neurons in experimental rats who underwent social interactions with either stressed or naive juveniles. The cell counting results suggested that activity in this tract increases during encounters with stressed juveniles in the posterior IC. To establish the necessity and sufficiency of this tract to the social affective preference behavior we chose to use a tract-specific chemogenetic approach (Jaramillo et al., 2018b). This required that we first demonstrate feasibility of chemogenetic manipulations in the SAP test. To this end, we virally expressed the inhibitory hM4Di receptor (Roth, 2016) in the IC and achieved inhibition via systemic administration of clozapine- $N$-oxide (CNO), which blocked the social preference for stressed juveniles and replicated our prior findings with pharmacological and optogenetic inhibition of IC (Rogers-Carter et al., 2018b). Following methods and considerations described by others (Stachniak et al., 2014; Smith et al., 2016; Mahler and Aston-Jones, 2018) to gain tract-specific, chemogenetic control of the IC $\rightarrow$ NAc circuit, we introduced hM4Di or hM3Dq in the IC under the synapsin promoter to achieve receptor expression in axon terminals in the NAc. CNO was delivered via bilateral cannula to the NAc, so that excitation or inhibition was selective to terminals of neurons that originate in IC, before testing. To determine whether IC $\rightarrow$ NAc neurons are necessary for social approach to stressed juveniles, we infused $\mathrm{CNO}$ before SAP tests in rats expressing hM4Di in IC $\rightarrow$ NAc neurons. This interfered with approach directed at stressed juveniles but not adults. Because social interaction with a stressed juvenile increased Fos in IC $\rightarrow$ NAc neurons, and chemogenetic inhibition reduced social interaction, we hypothesized that a gain-of-function in the IC $\rightarrow$ NAc tract would be sufficient to promote social interaction. To test this, $\mathrm{CNO}$ was infused in experimental rats with hM3Dq in the IC $\rightarrow$ NAc tract before 1 -on- 1 social interactions with either naive juvenile or naive adult conspecifics. To test whether the IC contributes during social reward per se, we adapted the SAP test to assess social novelty preference (Smith et al., 2018) in a task in which adult experimental rats preferred to investigate a novel socially rewarding adult conspecific compared with a familiar (cagemate) conspecific. Next, we inhibited the IC using hM4Di and systemic $\mathrm{CNO}$ and found that social novelty preference remained intact. A number of control, verification, and chemogenetic specificity experiments are also described to address important concerns that relate to the interpretation of chemogenetic experiments.

Subjects. Male Sprague Dawley rats were from Charles River Laboratories at either $250 \mathrm{~g}$ or PN22 or PN42. All rats were acclimated to the vivarium in the Boston College Animal Care facility for a minimum of $7 \mathrm{~d}$ before surgery or behavioral testing resulting in groups of experimental adult rats at PN60-PN80, juvenile conspecifics at PN30 d and adult, postpubescent conspecifics at PN50 at the time of testing. Subjects were housed in groups of 2 or 3, maintained on a $12 \mathrm{~h} \mathrm{light/dark} \mathrm{cycle,} \mathrm{and}$ provided food and water ad libitum. Behavioral testing occurred within the first $4 \mathrm{~h}$ of the light cycle. All procedures were approved by the Boston College Institution Animal Care and Use Committee and adhered to the National Institutes of Health's Guide for the Care and Use of Laboratory Animals.

Social affective preference tests. As previously reported (Rogers-Carter et al., 2018b), the SAP test allows for the quantification of social exploration directed at two novel conspecifics, one of which is stressed via foot shock and the other naive to stress treatment. On day 1 , experimental rats were acclimated to a plastic tub cage arena $(50 \mathrm{~L} \times 40 \mathrm{~W} \times 20 \mathrm{~cm} \mathrm{HH})$ containing $\beta$ chip bedding and a wire lid. On day 2 , the experimental rats were presented two naive conspecifics, either juvenile (PN28-PN32) or adult (PN50-PN54) pairs, which were held in clear acrylic chambers (18 $\mathrm{L} \times 21 \mathrm{~W} \times 10 \mathrm{~cm} \mathrm{H}$ ). Conspecific chambers were comprised of acrylic rods spaced $1 \mathrm{~cm}$ apart center to center (see Fig. 1C) and placed at opposing ends of the cage for a $5 \mathrm{~min}$ trial. The chambers were designed to be large enough to permit the contained rat to move freely. On days 3 and 4 , one conspecific was stressed via 2 brief foot shocks immediately before each test ( $5 \mathrm{~s}$ duration, $1 \mathrm{~mA}, 60 \mathrm{~s}$ intershock interval; Precision Regulated Animal Shocker; Coulbourn Instruments). SAP tests began when the chambers containing naive and stressed conspecifics were placed in the test arena. A trained observer blinded to treatment quantified the amount of social exploration initiated by the experimental rat, which included: nose-to-nose contact, nose-to-body contact, or reaching toward the conspecifics. Social exploration was quantified first during live testing and again from digital video recordings by a second observer who was blind to experimental conditions to establish interraterreliability. The correlation between observers for the SAP trials included in the current experiments was $r_{35}=0.922, R^{2}=0.849, p<0.0001$.

Social interaction. This procedure is used to quantify social exploration when an experimental test rat and a target conspecific were free to inter- 
act. On day 1, experimental rats were habituated to a standard plastic cage with $\beta$-chip bedding and wire lid for $1 \mathrm{~h}$. On day 2 , a juvenile or adult conspecific was introduced to the arena for $5 \mathrm{~min}$ and a trained observer quantified social interaction initiated by the experimental rat including pinning, sniffing and allogrooming. Exploration was quantified first during live testing and again by an observer blind to treatment from digital video recordings. The correlation between observers for the social interaction tests included in the current experiments was $r_{12}=$ $0.997, R^{2}=0.993, p<0.0001$.

Cannula placements and virus microinjections. Under inhaled isoflurane $\left(2-5 \% \mathrm{v} / \mathrm{v}\right.$ in $\left.\mathrm{O}_{2}\right)$, bilateral cannula (Plastics One) were implanted in the NAc (from bregma: A/P $+1.9 \mathrm{~mm}, \mathrm{M} / \mathrm{L}+/-1.8 \mathrm{~mm}, \mathrm{D} / \mathrm{V}-6.5$ $\mathrm{mm}$ ) and fixed in place with acrylic cement and stainless steel screws. For chemogenetic manipulations, $600 \mathrm{nl}$ of a viral vector containing either pAAV5-hSyn-hM4D(Gi)-mCherry (hM4Di; Addgene viral prep; catalog \#50475-AAV5; titer: $\left.9 \times 10^{12} \mathrm{GC} / \mathrm{mL}\right)$, pAAV5-hSyn-hM3D(Gq)mCherry (hM3Dq; Addgene viral prep; catalog \#50474-AAV5; titer: $4.8 \times 10^{12} \mathrm{GC} / \mathrm{mL}$ ), or pAAV5-hSyn-mCherry (mCherry; Addgene viral prep; catalog \#114472), were microinjected bilaterally at 2 sites in the posterior insular cortex (from bregma: $\mathrm{A} / \mathrm{P}-1.8 \mathrm{~mm}$ and $-1.6 \mathrm{~mm}, \mathrm{M} / \mathrm{L}$ $\pm 6.5 \mathrm{~mm}, \mathrm{D} / \mathrm{V}-6.9 \mathrm{~mm}$ ) at $100 \mathrm{nl} / \mathrm{min}$ and allowed $7 \mathrm{~min}$ for diffusion. These coordinates led to transduction within the posterior IC for consistency with our prior work and the results of the retrograde Fos counting experiment. In tract-specific chemogenetic manipulations, bilateral NAc cannulas were implanted during the same surgery as described above. This approach allowed tract-specific control by directly infusing the hM3Dq and hM4Di agonist CNO to the terminals of IC neurons in NAc (see pharmacological manipulations). For retrograde tracing, $300 \mathrm{nl}$ of cholera toxin B conjugated to Alexa Fluor 488 ( $\mathrm{CTb}^{488}$; Thermo Fisher, catalog \#C34775) were microinjected to the right hemisphere NAc as previously (Dong et al., 2017; Park et al., 2018) at a rate of $100 \mathrm{nl} / \mathrm{min}$ and allowed $4 \mathrm{~min}$ for diffusion. After surgery, rats were administered meloxicam $(1 \mathrm{mg} / \mathrm{kg}$, Eloxiject; Henry Schein) and the antibiotic penicillin (12,000 units, Combi-pen; Henry Schein) and allowed 2 to 3 weeks to recover before behavioral testing as described below.

Pharmacological manipulations. To reversibly inhibit NAc activity the voltage gated sodium channel blocker TTX (Tocris Bioscience) was dissolved in sterile $0.9 \%$ saline to a concentration of $10 \mu \mathrm{M}$. TTX or vehicle (sterile $0.9 \%$ saline) were infused bilaterally $(0.5 \mu \mathrm{l} /$ side $)$ to NAc $15 \mathrm{~min}$ before SAP testing (Christianson et al., 2011). For chemogenetic inhibition of IC cell bodies, experimental rats were weighed on the day of testing and given $0,0.3$ or $3 \mathrm{mg} / \mathrm{Kg} \mathrm{CNO}$ (Tocris Bioscience) dissolved in a vehicle of $10 \%$ DMSO and sterile $0.9 \%$ saline 45 min before SAP testing via intraperitoneal injection. For tract-specific experiments, $1 \mu \mathrm{M} \mathrm{CNO}$ in a vehicle of $0.1 \%$ DMSO and sterile $0.9 \%$ saline was infused through the NAc guide cannula $(0.5 \mu \mathrm{l} / \mathrm{side}) 30 \mathrm{~min}$ before testing as in Mahler et al. (2014).

Tissue collection and immunohistochemistry. Immediately after testing, rats from TTX experiments were overdosed with tribromoethanol (Fisher Scientific), decapitated, brains were dissected and flash frozen in 2-methylbutane (Fisher Scientific). All other rats were overdosed with tribromoethanol and perfused with cold $0.01 \mathrm{~m}$ heparinized PBS followed by $4 \%$ paraformaldehyde as described previously (Rogers-Carter et al., 2018b). Dissected brains were stored at $4^{\circ} \mathrm{C}$ in $4 \%$ paraformaldehyde for $24 \mathrm{~h}$ and transferred to $30 \%$ sucrose for $2 \mathrm{~d}$. $40 \mu \mathrm{m}$ coronal sections were cut on a freezing cryostat (Leica). Alternating NAc sections were either directly mounted to gelatin subbed slides and stained with cresyl violet for cannula tip verification or stored in cryoprotectant as free-floating sections for immunostaining. IC sections were directly mounted to slides and coverslipped with Vectashield (Vector Laboratories) to confirm virus transduction. To visualize Fos or mCherry fibers in $\mathrm{NAc}$, floating sections were quenched for endogenous enzymes with $3 \%$ $\mathrm{H}_{2} \mathrm{O}_{2}$ and blocked with $2 \%$ normal donkey serum (Jackson ImmunoResearch) in PBS-T (0.01\% Triton X-100) and then incubated overnight in rabbit anti-c-Fos primary antibody (1:5000; Millipore; catalog \#ABE457; lot \#2987437) or the rabbit anti-mCherry primary antibody (1:200; Invitrogen; catalog \#PA5-34974; lot \#114936) for $2 \mathrm{~h}$. Next, sections were washed in PBS-T and incubated in biotinylated donkey-anti-rabbit secondary antibody (1:200; Jackson ImmunoResearch; catalog \#711-035-
152) followed by the avidin-biotin complex kit (ABC Elite Kit; Vector Laboratories) and visualized with chromogen precipitate (NovaRed; Vector Laboratories). Stained sections were mounted on slides, dehydrated, cleared and coverslipped with Permount (Fisher Scientific). For Fos counting, tiled images of NAc tissue were acquired using a Zeiss AxioCam HRc digital camera through a $10 \times$ objective (numerical aperture [NA] 0.45) and Fos-immunoreactive cells were manually counted in ImageJ by an observer blind to treatment. For tissue containing $\mathrm{CTb}^{488}$, floating sections were washed in PBS-T, blocked in 5\% normal goat serum in PBS-T, and incubated overnight in rabbit-anti-c-Fos primary antibody as above at $4{ }^{\circ} \mathrm{C}$, washed, incubated in the Dylight 549 goat-antirabbit secondary antibody (1:500; Vector Laboratories; catalog \#DI1549), floated on slides, and coverslipped with Vectashield. For cell counting, green $\left(\mathrm{CTb}^{488}\right)$ and red (Fos) channel tiled images of NAc were acquired using a Hamamatsu digital camera through a 10x objective (NA 0.45) and CTb ${ }^{488}$ and Fos-positive colabeled cells were manually counted in Image by a trained observer blinded to treatment. Cells were counted within a $1250 \mu \mathrm{m}^{2}$ area in IC and from a $350 \mu \mathrm{m}^{2}$ area in NAc.

Electrophysiology. To verify the efficacy of hM4di and hM3Dq on neural activity whole-cell recordings were made in $300 \mu \mathrm{m}$ thick coronal sections taken from a subset of rats at the conclusion of the behavioral experiments. Slice preparation, internal and external solutions, patchclamp electrodes and current-clamp experiments and data analysis were conducted as previously (Rogers-Carter et al., 2018b). Briefly, whole-cell recordings were achieved in cells visually identified as mCherry positive. Intrinsic excitability was established by quantifying the number of action potentials generated by a small depolarizing current injection (rheobase) in normal artificial CSF, during application of CNO $(10 \mu \mathrm{M}$ as in Venniro et al., 2017) and for an additional 10 min with CNO in the bath.

Data analysis. The initial sample sizes for these experiments were determined by a priori power analysis $\left(G^{\star}\right.$ Power ver. 3.1$)$ using the effect sizes from our prior observations using repeated-measures designs. The effect size for data relating to the significant interaction in Figures $1 C$ and 2 of Rogers-Carter et al. (2018a) were $\eta_{p}^{2}=0.71(n=20$, mixed-model between-subjects and within-subjects factors) and $\eta_{P}^{2}=0.67(n=18$, within-subjects factors only), respectively. To achieve power $=0.80$ to detect a similar large effect size for a two-way interaction in a withinsubjects model would require $n=8$. Here, we set out to meet or exceed these numbers by assigning a minimum of eight subjects to each treatment condition. All data were included unless cannula implants or virus transduction missed the target site. Experimental treatments were counterbalanced in order and repeated-measures designs were used except when noted. We tested for order effects in pilot studies and did not find evidence of treatment order effects on SAP behavior when using tetrodotoxin or $\mathrm{CNO}$ injections (either intraperitoneal or intracranial administration). Before analysis, all data were visually inspected for normality and order effects. In all cases, the data were deemed suitable for parametric analysis and all data are depicted in the figures. Social exploration times during SAP tests were compared using a two-way ANOVA with conspecific stress (naive or stressed) and drug treatment (vehicle or drug) analyzed as within-subjects factors. To control for individual variation in sociality, exploration times were converted to a preference score, which was computed as the percentage of total social exploration time directed toward the stressed conspecific and analyzed with $t$ test or twoway ANOVA, depending on experimental design. $t$ tests were used to compare social exploration in rats sacrificed for postmortem CTb, Fos, and $\mathrm{CTb}+$ Fos counts, which were analyzed with mixed-model ANOVAs with insula ROI as a repeated measure and conspecific stress as a between-subject factor. All analyses were conducted in Prism 8.2 (GraphPad). Significant main effects and interactions were followed with Sidak post hoc comparisons to control experimentwise type 1 errors to $p<0.05$. Effect sizes are reported as $\eta^{2}$ for $t$ tests or $\eta_{p}^{2}$ for main effects and interactions in ANOVAs.

\section{Results}

Pharmacological inactivation of NAc abolished preference for stressed PN30, but not PN50, conspecifics

To test if NAc was necessary for SAP behavior, experimental rats were implanted with bilateral NAc cannula (Fig. $1 A, B$ ), allowed 1 
A

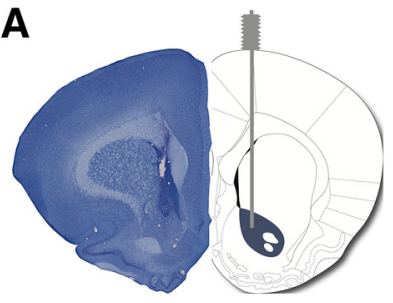

B

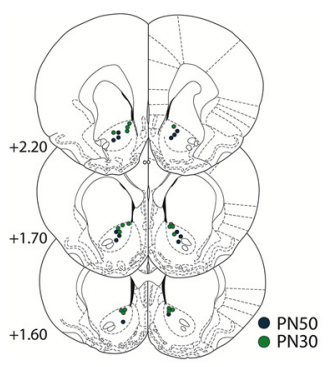

C

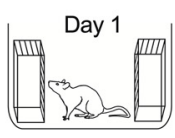

arena habituation

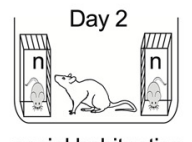

social habituation

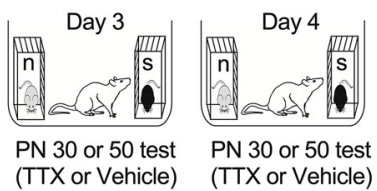

D
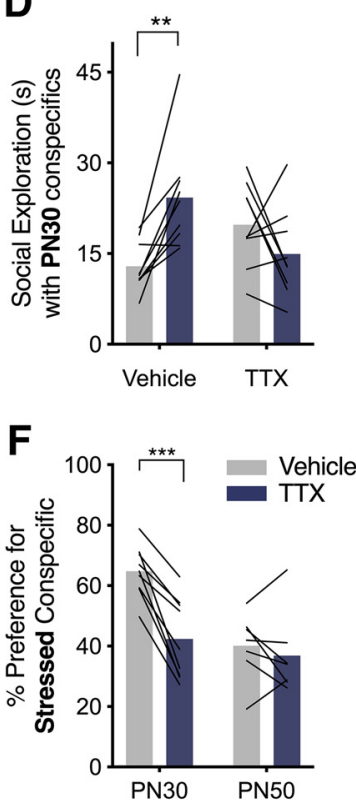

Figure 1. Pharmacological inhibition of the NAc abolished the social affective preference for stressed PN30, but not PN50, conspecifics. $A$, Representative image of a cannula tract in NAC (left) and corresponding rat brain atlas diagram (right). $\boldsymbol{B}$, Map of bilateral cannula tip placements in NAc from experimental rats tested with PN30 (green; $n=9$ ) and PN50 (blue; $n=7$ ) conspecifics. C, Diagram of SAP test procedure with naive (n) and stressed (s) conspecifics. D, Mean (with individual replicates) time spent exploring the naive and stressed PN30 conspecifics during the $5 \mathrm{~min}$ trial. Vehicle-treated rats preferred to explore stressed PN30 conspecifics compared with naive PN30 conspecifics ( $p=0.006)$, which was abolished via bilateral infusion ofTTX ( $100 \mathrm{~nm}, 0.5 \mu \mathrm{l} / \mathrm{side}$ ) in NAc $15 \mathrm{~min}$ before testing $(p=0.203)$. $\boldsymbol{E}$, Mean (with individual replicates) time spent exploring the naive and stressed PN50 conspecifics during the 5 min trial. Both vehicle-treated ( $p=0.031$ ) and TTX-treated $(p=0.020)$ rats preferred to explore naive PN50 conspecifics compared with the stressed PN50 conspecifics. $\boldsymbol{F}$, Mean (with individual replicates) data from $\boldsymbol{D}$ and $\boldsymbol{E}$ shown as the percentage of total social exploration time that was spent investigating the stressed conspecific. Rats tested with PN30 conspecifics show preference (as indicated by scores $>50 \%$ ) for the stressed conspecific under vehicle treatment, which was significantly reduced after pharmacological inactivation of NAc with TTX ( $p=0.0001$ ). Rats tested with PN50 conspecifics show a preference for naive conspecifics, which was unaffected by TTX treatment $(p=0.452)$. ${ }^{*} p<0.05,{ }^{* *} p<0.01,{ }^{* * *} p<0.001$.

week of recovery, and then underwent SAP testing with either PN30 $(n=9)$ or PN50 $(n=7)$ conspecifics. Experimental rats were tested after vehicle and drug injections on days 3 and 4 of testing and the amount of time the experimental rat explored each conspecific was recorded; treatment order was counterbalance (Fig. 1C). For rats tested with PN30 conspecifics, we report a significant conspecific stress $\times$ drug interaction $\left(F_{(1,8)}=18.49\right.$; $\left.p=0.003, \eta_{P}^{2}=0.26\right)$. After vehicle treatment, rats tested with
PN30 conspecifics showed a preference to explore the stressed conspecific over the naive ( $p=0.006)$, which was abolished after bilateral pharmacological inactivation of NAc ( $p=0.203$; Fig. $1 D)$. For rats tested with PN50 conspecifics, a two-way ANOVA found a main effect of conspecific affect $\left(F_{(1,6)}=5.45, p=0.058\right.$, $\left.\eta_{P}^{2}=0.30\right)$, which confirmed replication our previous findings (Rogers-Carter et al., 2018a,b) that experimental rats prefer to explore the naive conspecific over the stressed after vehicle treatment ( $p=0.031)$, which was also maintained after TTX treatment ( $p=0.020$; Fig. $1 E)$. To compare the effect of TTX in NAc between age experiments, social exploration times were converted to percentage preference scores and shown in Figure $1 F$ for comparison. Here, there were significant main effects of conspecific age $\left(\mathrm{F} 1,14=8.192, p=0.012, \eta_{P}^{2}=0.22\right), \operatorname{drug}\left(F_{(1,14)}=\right.$ 25.31, $\left.p<0.001, \eta_{P}^{2}=0.16\right)$ and a significant conspecific age $\times$ drug interaction $\left(F_{(1,14)}=14.09, p=0.002, \eta_{p}^{2}=0.09\right)$, which reveals that preference scores from experimental rats tested with PN30, but not PN50 conspecifics, are sensitive to TTX treatment. Preference scores from experimental rats tested with PN30 conspecifics were significantly greater in vehicle trials than TTX trials $(p<0.0001)$. However, TTX did not alter preference scores in experimental rats tested with PN50 conspecifics $(p=0.651)$. This reflects that avoidance of stressed PN50 conspecifics was maintained after TTX treatment (Fig. $1 F$ ). These analyses demonstrate that the NAc is necessary for experimental rats' preference to explore stressed PN30 but did not influence interactions with either stressed or naive PN50, conspecifics.

\section{Exposure to stressed PN30 conspecifics activated IC neurons that project to NAc}

The NAc is a major target of IC afferents (Wright and Groenewegen, 1996) and the foregoing results suggest that these two structures are implicated in control of social responses to stressed juvenile conspecifics. Thus, we predicted that interaction with a stressed PN30 conspecific would activate NAc projecting IC neurons. To test this hypothesis, 12 experimental rats received unilateral microinjections of the retrograde tracer $\mathrm{CTb}^{488}$ in the left hemisphere NAc (Fig. 2A,B) as previously (Dong et al., 2017; Park et al., 2018) so that neurons that project from IC to NAc can be identified for cell counting (Fig. 2E). 2 weeks after surgery, in a between-subjects design, six experimental rats underwent social interactions with naive PN30 conspecifics and six underwent interactions with stressed PN30 conspecifics (Fig. 2C). As previously reported (Rogers-Carter et al., 2018b), experimental rats displayed higher levels of social exploration with stressed PN30 conspecifics compared with naive PN30 conspecifics $\left(t_{(10)}=\right.$ $3.281, p=0.008, \eta^{2}=0.52$; Fig. $2 D$ ). Rats were euthanized 90 min after testing and tissue was processed and stained for fluorescent Fos expression. Tiled images of the IC ipsilateral to the NAc injection site were acquired at the following IC locations relative to bregma: +2.52 and $0 \mathrm{~mm}$ (anterior IC), $-0.24 \mathrm{~mm}$ and $-0.72 \mathrm{~mm}$ (medial IC) and -1.72 and -2.04 (posterior IC) so that the number of Fos-positive, $\mathrm{CTb}^{488}$-positive, and colabeled neurons could be counted across the rostrocaudal extent of IC. All cells were counted in a field of view with a fixed area across all ROIs. Before conducting analysis, brains were screened for evidence of $\mathrm{CTb}^{488}$ deposits localized to the NAc (Fig. $2 B$ ). Four animals, two from each stress group, did not exhibit $\mathrm{CTb}^{488}$ in the NAc and so were excluded from all subsequent analysis. We were unable to obtain counts from one subject from each treatment group at the anterior IC and medial IC regions resulting in $n=3$ or 4 observations per group. All individual replicates are depicted in Figure 2, $F-H$. To establish whether the resulting 
A

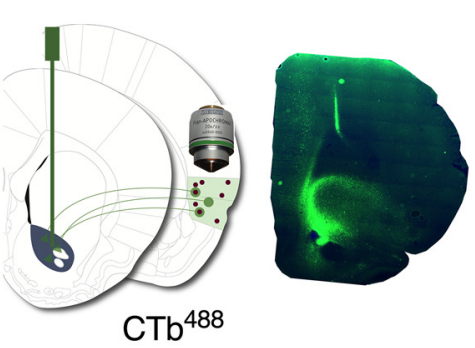

B

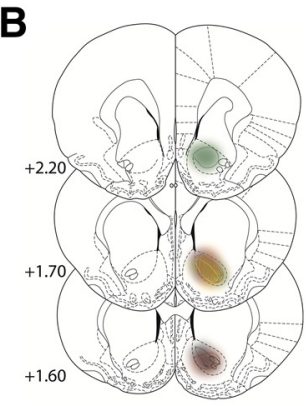

C

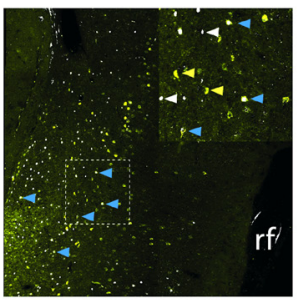

D

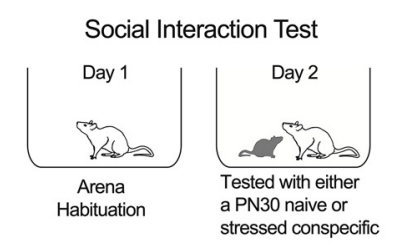

G

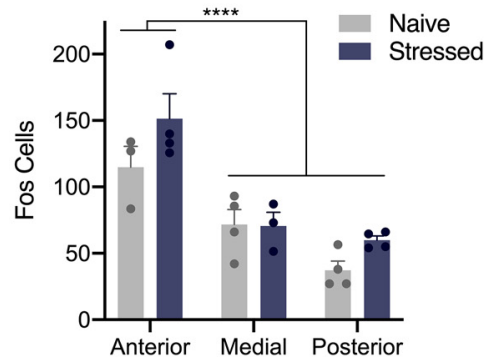

E

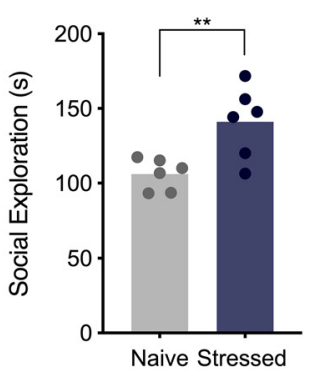

F

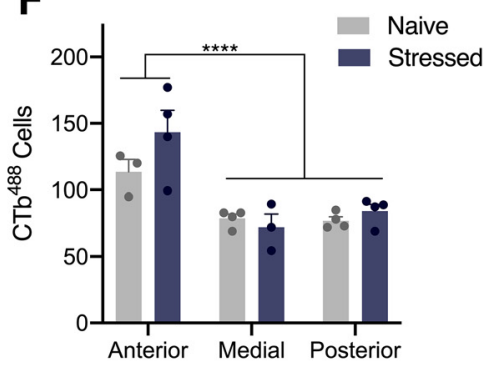

H

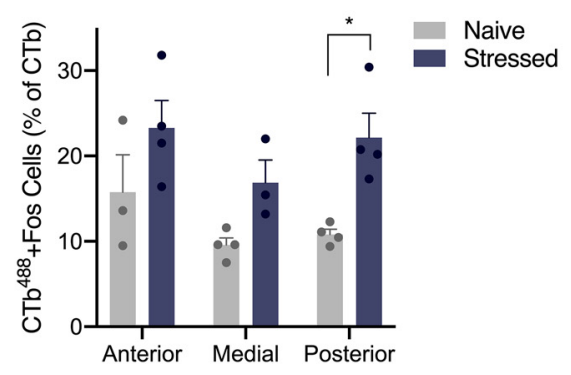

Figure 2. Exposure to stressed PN30 conspecifics elicited greater Fos immunoreactivity in IC $\rightarrow$ NAc neurons than exposure to naive PN30 conspecifics. $\boldsymbol{A}$, Schematic depicting unilateral $300 \mathrm{nl}$ injections of the retrograde tracer $\mathrm{CTb}^{488}$ in the right hemisphere NAc (left) and representative image of $\mathrm{CTb}^{488}$ in NAc (right: green $=\mathrm{CTb}^{488}$, blue $=\mathrm{DAPI}^{4}$. $\boldsymbol{B}$, CTb ${ }^{488}$ expression in NAc: each experimental rat is represented in a different color. $C, \mathrm{CTb}^{488}$ - and Fos-positive neurons in IC. Image is psuedocolored; white indicates Fos, yellow is $\mathrm{CTb}^{488}$, blue is colabeled cells. rf, Rhinal fissure. $\boldsymbol{D}$, Diagram of behavioral procedures. Six experimental rats explored naive PN30 conspecifics and six explored stressed PN30 conspecifics. $\boldsymbol{E}$, Mean (with individual values) social exploration time. Experimental rats presented stressed PN30 conspecifics engaged in more social exploration than rats presented naive PN30 conspecifics ( $p=0.008)$. $\boldsymbol{F}$, Counts of CTb ${ }^{488}$-positive cells in anterior, medial, and posterior IC. More CTb ${ }^{488}$-positive cells were observed in anterior IC than medial or posterior IC ( $\left.p<0.0001\right)$; there was no effect of conspecific stress on the number of CTb ${ }^{488}$-positive cells at any ROI. G, Counts of Fos-positive cells in each IC region. More Fos-positive cells were observed in anterior IC than medial or posterior ( $p<0.0001$ ); there was no effect of conspecific stress on the number of Fos-positive cells at any ROI. $\boldsymbol{H}$, Counts of colabeled CTb ${ }^{488}$ and Fos-positive neurons. A main effect of stress was observed ( $\left.p=0.0008\right)$, and experimental rats that explored stressed PN30 conspecifics expressed more Fos immunoreactivity in $\mathrm{CTb}^{488}$-positive neurons in posterior IC $(p=0.015)$ compared with experimental rats who explored naive PN30 conspecifics. ${ }^{*} p<0.05,{ }^{* *} p<0.01,{ }^{* * *} p<0.0001$.

small sample size was sufficiently powered for our experimental questions, we used a mixed-effects two-way ANOVA with ROI and conspecific stress as within-subjects factors to compare $\mathrm{CTb}^{488}$ expression across anterior, medial, and posterior IC. It is established that the insula projections to NAc is most dense in the anterior IC, compared with the posterior IC (Parks et al., 2015) and this trend was evident and supported by a significant main effect of ROI $\left(F_{(2,16)}=19.3, p<0.0001\right)$. Anterior IC had significantly more $\mathrm{CTb}^{488}$-positive cells than medial or posterior IC $(p<0.001$; Fig. $2 F)$. Proceeding to the Fos analyses, a 2-way ANOVA revealed a main effect of ROI $\left(F_{(2,10)}=30.53, p<\right.$ $0.0001)$. More Fos-positive cells were found in anterior IC, than medial or posterior IC (ps $<0.001$; Fig. $2 G$ ) and there were no significant differences in Fos between conspecific stress groups. Regarding $\mathrm{CTb}^{488}$ and Fos-positive co-labeled neurons, we report a main effect of Conspecific Stress $\left(F_{(1,16)}=16.89, p=\right.$ $0.0008)$. Although the trend of greater $\mathrm{CTb}^{488}$ and Fos-positive was evident across ROIs, the only significant post hoc comparison was observed between naive and stressed conspecific groups in the posterior IC $(p=0.015$; Fig. $2 H)$. In sum, exposure to stressed conspecifics led to greater activity in NAc-projecting IC neurons with the greatest differential activation observed in the posterior subregion of IC.

\section{Chemogenetic inhibition of IC blocked social affective preference behavior}

We previously reported (Rogers-Carter et al., 2018b) that the preference to explore stressed PN30 and naive PN50 conspecifics was mediated by IC using an optogenetic approach. Here, an intersectional chemogenetic approach was preferred as the manipulation because it is less restrictive to movement, well suited to control neural activity in social behavior, and has been used to investigate IC projections to NAc and central amygdala in different paradigms (Venniro et al., 2017; Jaramillo et al., 2018a). Therefore, we first sought to replicate our prior finding and establish feasibility of chemogenetic control of IC neurons by using the inhibitory chemogenetic hM4Di receptor expressed in neurons under control of the synapsin promoter. Experimental rats ( $n=10)$ were bilaterally transduced with pAAV5-hSynhM4D(Gi)-mCherry in IC (Fig. 3A,B). Two weeks later, rats 
underwent the SAP test procedure with PN30 conspecifics after injections of vehicle or $\mathrm{CNO}(3 \mathrm{mg} / \mathrm{kg}$, i.p.), order counterbalanced, $45 \mathrm{~min}$ before testing (Fig. $3 D, F)$. This produced a significant conspecific stress $\times$ drug interaction $\left(F_{(1,9)}=\right.$ $\left.34.22, p=0.0002, \eta_{P}^{2}=0.23\right)$. Vehicletreated rats spent more time exploring stressed PN30 conspecifics than naive PN30 conspecifics ( $p=0.0001$; Fig. $3 F)$. This preference was abolished after inhibition of IC neurons as experimental rats tested with $\mathrm{CNO}$ did not show a preference for the naive or stressed PN30 conspecific ( $p=0.517$; Fig. $3 F)$. To interpret the effect of $\mathrm{CNO}$ in behavioral experiments, a number of factors must be considered (MacLaren et al., 2016; Mahler and Aston-Jones, 2018; Manvich et al., 2018). To confirm these results were not an effect of $\mathrm{CNO}$ alone, experimental rats without virus transduction $(n=8$; Fig. $3 E$ ) underwent the SAP procedure after intraperitoneal $\mathrm{CNO}$ injections at the following doses: $0,0.3$, and $3.0 \mathrm{mg} / \mathrm{kg}$, the latter two of which have been previously reported to effectively modulate neurons transduced with chemogenetic viruses (Roth, 2016). A two-way ANOVA with conspecific stress and dose as withinsubjects factors revealed a main effect of conspecific stress $\left(F_{(1,7)}=62.93, p=\right.$ $\left.0.0001, \eta_{p}^{2}=0.45\right)$. Post hoc comparisons further revealed that, at each dose, experimental rats spent more time exploring the stressed PN30 conspecifics than the naive $(0 \mathrm{mg} / \mathrm{kg}, p=0.0009 ; 0.3 \mathrm{mg} / \mathrm{kg}$, $p=0.0036 ; 3.0 \mathrm{mg} / \mathrm{kg}, p=0.0063)$. These findings demonstrate that i.p. injections of CNO at 0.3 or $3.0 \mathrm{mg} / \mathrm{kg}$ per se did not interfere with social affective preference behavior (Fig. 3G,H). To demonstrate virus efficacy, a cohort of experimental rats $(n=9)$ was bilaterally transduced with the sham virus pAAV5-hSyn-mCherry in IC (Fig. 3C), and 2 weeks later underwent SAP testing after vehicle and $3.0 \mathrm{mg} / \mathrm{kg}$ intraperitoneal $\mathrm{CNO}$ injections (order counterbalanced; Fig. 3E, right). Rats spent more time exploring the stressed PN30 conspecific after both vehicle $(p=$ $0.003)$ and $\mathrm{CNO}(p=0.002)$ treatment (Fig. $3 F$ ), which supports the main finding that IC inhibition achieved by chemogenetic inhibition blocked the social affective preference for the stressed PN30 conspecific and established feasibility for chemogenetic control of IC neurons during SAP testing. For side-by-side comparison of the effect of $\mathrm{CNO}$ in the sham and virus preparations, the data in Figure $3, E$ and $F$, were converted to percentage preference scores and presented side by side for comparison in Figure $3 \mathrm{H}$. In no instance did administration of CNO alone $\left(F_{(7,14)}=0.343, p=\right.$ $0.956, \eta^{2}<0.01$ ), or vehicle or CNO administration to rats with the sham mCherry virus $\left(t_{(8)}=0.010, p=0.992, h^{2}<0.01\right)$

D

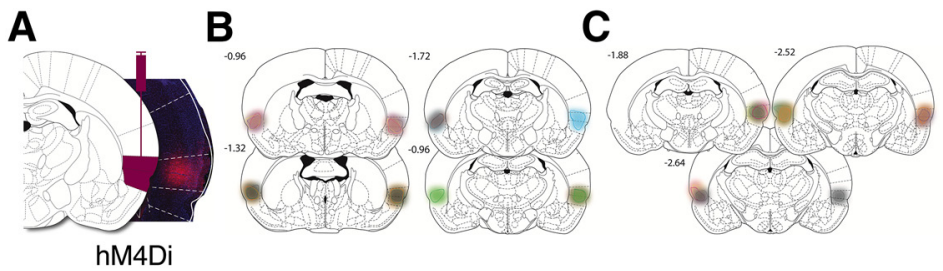

E
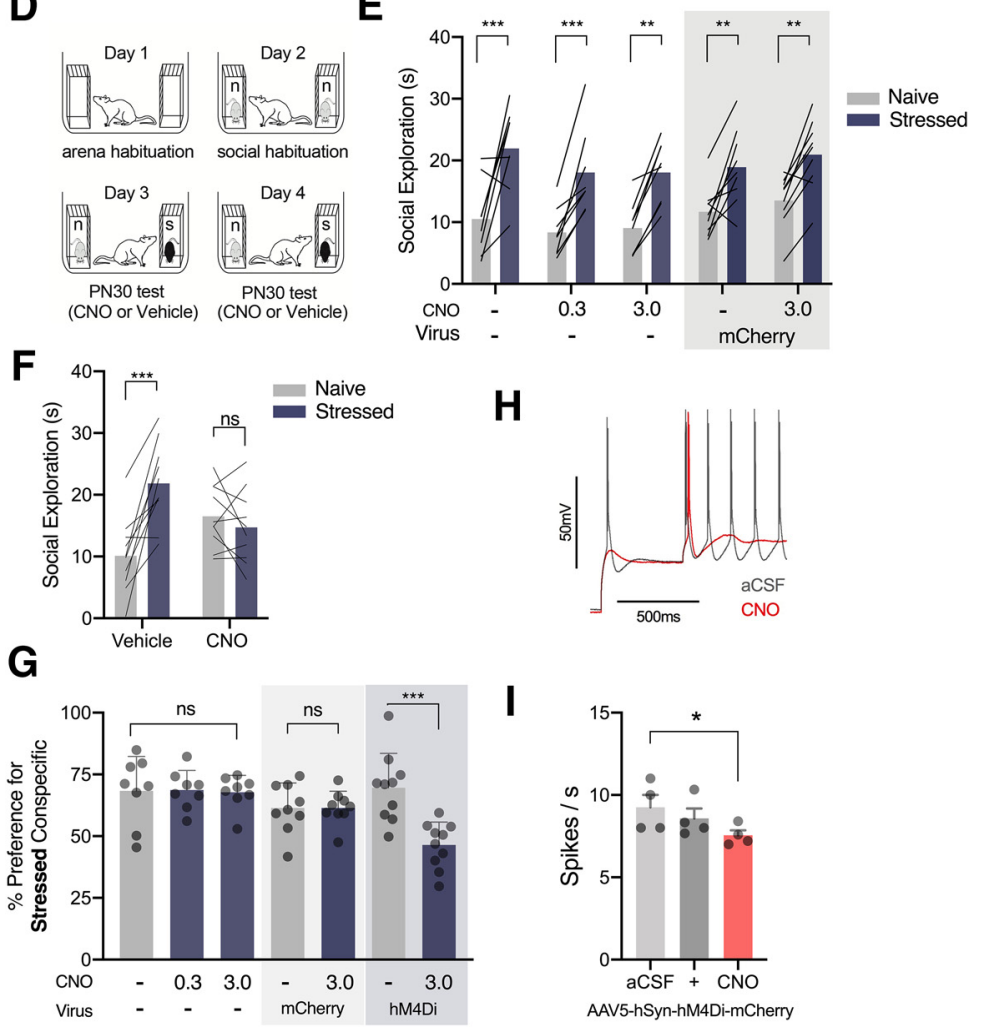

Figure 3. Chemogenetic inhibition of IC blocked the social affective preference for stressed PN30 conspecifics. $\boldsymbol{A}$, Representative image of pAAV5-hSyn-hM4D(Gi)-mCherry transduction in IC (right) and corresponding rat brain atlas diagram (left). B, Map of bilateral IC pAAV5-hSyn-hM4D(Gi)-mCherry expression in experimental rats. Each subject is represented in a different color $(n=$ 10); bold outlines represent the greatest intensity of mCherry expression and the corresponding faded overlay depicts the full extent of virus transduction. C, Map of bilateral IC sham pAAV5-hSyn-mCherry expression, $n=8$. D, Diagram of SAP test procedure. $\boldsymbol{E}$, Control experiments: Experimental rats $(n=8)$ without viral transduction underwent the SAP test procedure $45 \mathrm{~min}$ after CNO injections at 3 doses: $0,0.3$, and $3.0 \mathrm{mg} / \mathrm{kg}$ (i.p.). No dose of CN0 disrupted the preference for the stressed PN30 conspecific ( 0 $\mathrm{mg} / \mathrm{kg}, p=0.0009 ; 0.3 \mathrm{mg} / \mathrm{kg}, p=0.0036 \mathrm{mg} / \mathrm{kg} ; 3 \mathrm{mg} / \mathrm{kg}, p=0.0063)$. Experimental rats transduced with a sham virus $(n=$ 9) preferred to explore the stressed PN30 conspecific under vehicle $(p=0.003)$ and $3 \mathrm{mg} / \mathrm{kg}$ CNO (i.p.) $(p=0.002)$. F, Mean (with individual replicates) time spent exploring the naive and stressed PN30 conspecifics during the 5 min trial. Vehicle-treated rats spent more time exploring the stressed PN30 conspecifics than the naive PN30 ( $p=0.0001)$, which was abolished in trials after injections of CNO (3 mg/kg, i.p.) $45 \mathrm{~min}$ before testing ( $p=0.517)$. $\boldsymbol{G}$, Data from $\boldsymbol{F}$ and $\boldsymbol{G}$ converted to percentage preference for the stressed conspecific. The only condition in which CNO reduced the preference for the stressed PN30 conspecific was in experimental rats transduced with hM4D(Gi) $(p=0.0003)$. $\boldsymbol{H}$, Representative acute, whole-cell recording of hM4D(Gi)-expressing IC neuron before and after bath application of CNO. I, After 10 min of bath application, CNO reduced the number of action potentials evoked by current injection in hM4D(Gi)-mCherry-positive neurons in the IC. $(+)$ indicates the beginning of CNO perfusion. (ns) indicates non significant difference. ${ }^{*} p<0.5,{ }^{* *} p<0.01,{ }^{* * *} p<0.001$.

disrupt the baseline preference to explore the stressed PN30 conspecific. In rats with transduced with hM4Di, administration of $\mathrm{CNO}$ attenuated the preference for the stressed conspecific $\left(t_{(9)}\right.$ $=5.654, p=0.0003, \eta^{2}=0.78$ ). Last, a subset of rats expressing hM4Di in the IC were killed for acute slice whole-cell recordings ( $n=4$ cells; Fig. $3 H, I$ ) as described previously (Rogers-Carter et al., 2018b). After following the methods described by Venniro et al. (2017), bath application of $10 \mu \mathrm{M}$ CNO reliably hyperpolarized and reduced excitability of IC neurons visually identified as mCherry-positive at the time of recording. A one-way repeatedmeasures ANOVA revealed a main effect of $\mathrm{CNO}\left(F_{(2,6)}=6.038\right.$, 
A

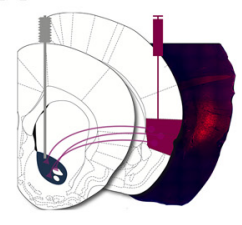

D

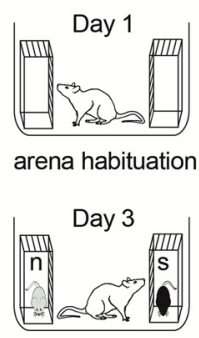

PN30 test

(CNO or Vehicle)

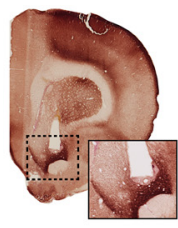

B

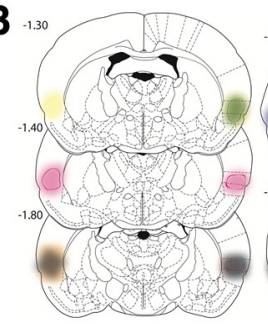

E

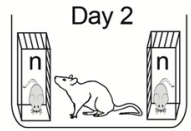

social habituation

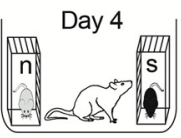

PN30 test

(CNO or Vehicle)
G

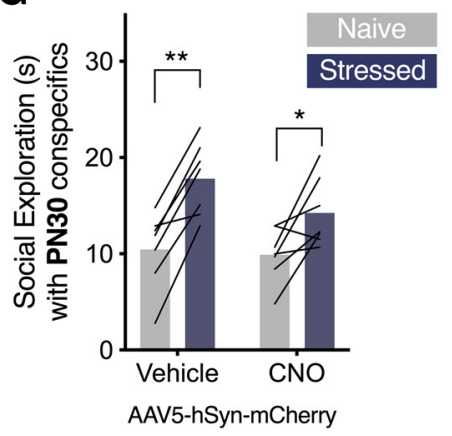

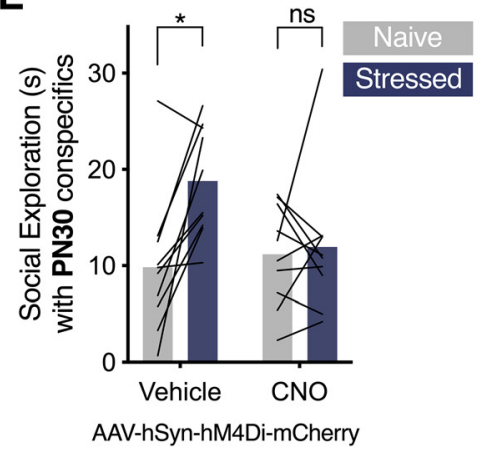

H

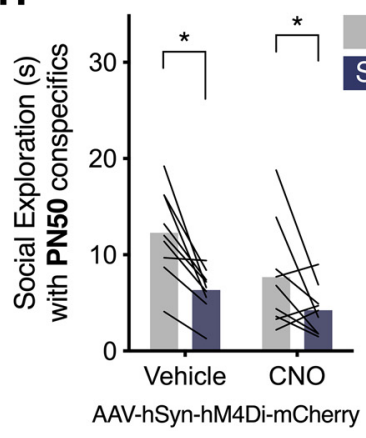

C

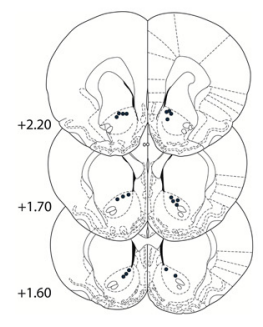

F

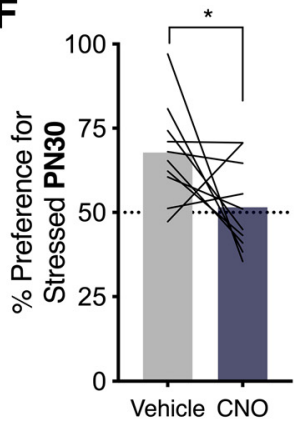

I

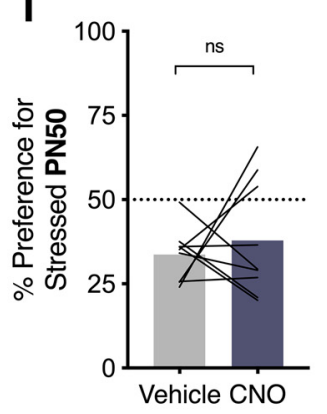

Figure 4. Chemogenetic inhibition of $\mathrm{IC} \rightarrow$ NAc terminals blocked the social affective preference for stressed PN30 conspecifics. A, Schematic of IC neurons transduced with pAAV5-hSynhM4D(Gi)-mCherry in IC and cannula implanted in NAc and representative image of virus transduction (left, red = native mCherry, blue $=$ DAPI) and verification of cannula tip in NAc with terminal fiber expression (right, mCherry immunoreactivity). $\boldsymbol{B}$, Summary of mCherry expression in IC $(n=10)$; bold outlines represent the region of maximum mCherry expression and the corresponding faded overlay depicts the full extent of virus transduction. C, Summary map of cannula tip placements in NAc. D, Diagram of behavioral procedures; treatment order on days 3 and 4 was counterbalanced. $\boldsymbol{E}$, Mean (with individual replicates, $n=10$ ) time spent exploring the naive and stressed PN30 conspecifics during the 5 min SAP test. Vehicle-treated rats spent more time exploring the stressed PN30 conspecifics than the naive PN30 conspecifics ( $p=0.023$ ), which was abolished via $1 \mu \mathrm{M}$ CNO injections 45 min before testing through the guide cannula. $F$, Data from $\boldsymbol{E}$ presented as the percentage preference for the stressed conspecific. Experimental rats show a preference to explore the stressed conspecific under vehicle treatment, which was significantly reduced after pharmacological inhibition of IC terminals in NAc in CNO $(p=0.033)$. G, To control for nonspecific effects of CNO in the NAc, a separate cohort of sham experimental rats without $\mathrm{hM} 4 \mathrm{D}$ (Gi) transduction underwent the SAP test. Under vehicle treatment, rats preferred to explore the stressed PN30 conspecifics $(p=0.003)$, which was maintained after microinfusion of $1 \mu \mathrm{M}$ CNO to the NAc 45 min before testing (0.031). $\boldsymbol{H}$, Mean (with individual replicates, $n=9$ ) time spent exploring the naive and stressed PN50 conspecifics during the 5 min SAP test after vehicle or 1 $\mu \mathrm{M}$ CNO injections. Test rats spent more time exploring the naive conspecifics than the stressed PN50 conspecifics regardless of injection (main effect of stress, $p=0.003$ ). $\boldsymbol{I}, \boldsymbol{D a t a}$ from $\boldsymbol{H}$ presented as the percentage preference for the stressed conspecific; scores did not differ between vehicle and drug groups. (ns) indicates non significant difference. ${ }^{*} p<0.05$, ${ }^{* *} p<0.01$.

$\left.p=0.040, \eta^{2}=0.66\right)$ and significantly fewer spikes under $\mathrm{CNO}$ compared with aCSF $(p=0.029)$.

\section{Chemogenetic inhibition of IC terminals in NAc blocked the preference for the stressed PN30 conspecifics}

To determine whether the IC $\rightarrow$ NAc pathway is necessary for SAP behavior with PN30 conspecifics, 10 experimental rats were transduced with the inhibitory hM4Di receptor via bilateral injection of pAAV5-hSyn-hM4D(Gi)-mCherry. Bilateral cannula were placed in NAc so that $\mathrm{CNO}$ could be directly administered to IC terminals (Fig. 4A, left). Rats were only included in the final analysis if there was positive evidence of virus expression in IC as in Figure $4 A$, right; map of virus expression in Figure $4 B$, bilateral cannula tip placement in NAc (Fig. $4 A$, right, map in Fig. $4 C$ ), and mCherry immunoreactivity of putative IC axon terminals in NAc (Fig. $4 A$, right inset). Two experimental rats were excluded due to lack of virus expression. 3 weeks after testing, experimental rats underwent the SAP procedure with PN30 conspecifics after microinjections of vehicle or $\mathrm{CNO}$ in NAc 15 min before testing (order counterbalanced; Fig. 4D,E). A 2-way ANOVA of social exploration time revealed a main effect of Conspecific Stress $\left(F_{(1,9)}=16.28, p=0.003, \eta_{p}^{2}=0.12\right)$; an interaction between conspecific stress and drug approached significance $\left(F_{(1,9)}=\right.$ $\left.4.214, p=0.070, \eta_{P}^{2}=0.09\right)$. In the vehicle condition, experimental rats preferred to explore the stressed PN30 conspecific over the naive ( $p=0.023)$. In the $\mathrm{CNO}$ condition, experimental rats did not exhibit preference for either the naive or stressed conspecific ( $p=0.959$; Fig. $4 E)$. When social exploration was converted to percentage preference scores (Fig. $4 F$ ), experimental rats showed scores that reflected more time spent with the stressed conspecific after vehicle injections, which was reduced by 
$\mathrm{CNO}\left(t_{(9)}=2.089, p=0.033, \eta_{P}^{2}=0.33\right)$. To rule out off-target effects of $\mathrm{CNO}$, a separate cohort of experimental rats without any virus administration was bilaterally implanted with guide cannula in NAc and underwent SAP testing with PN30 conspecifics. One trial followed microinjections of vehicle solution, and the other followed microinjections of CNO. A two-way ANOVA revealed a main effect of drug $\left(F_{(1,6)}=15.48, p=0.008, \eta_{P}^{2}=\right.$ $0.04)$ and conspecific stress $\left(F_{(1,6)}=34.78, p=0.001, \eta_{P}^{2}=0.40\right)$. Experimental rats preferred to explore the stressed PN30 conspecific in both the vehicle $(p=0.003)$ and $\mathrm{CNO}(p=0.031)$ conditions (Fig. $4 G$ ), which supports that the effect of $\mathrm{CNO}$ observed in hM4Di expressing rats cannot be attributed to off target effects of $\mathrm{CNO}$ on NAc function. To test whether IC $\rightarrow$ NAc neurons contribute to social avoidance of stressed adults, a separate cohort of rats received hM4D(Gi) injections in the IC and cannula placements in the NAc as above. Every aspect of the experiment was the same as in Fig. $4 D$ and $E$ except that the conspecifics were PN50 adult rats. Only rats meeting the criteria for IC hM4D(Gi) expression, bilateral NAc cannula placement, mCherry expression in NAc, and avoidance of the stressed conspecific under vehicle conditions were included in analysis. In adult SAP tests, test rats spent more time interacting with the naive conspecific after both vehicle and drug conditions. A two-way ANOVA revealed a main effect of conspecific stress $\left(F_{(1,8)}=16.77, p=\right.$ $\left.0.003, \eta_{p}^{2}=0.24\right)$. As in the CNO only experiment, there was a small but significant effect of $\mathrm{CNO}$ on overall interaction levels evinced by a main effect of drug $\left(F_{(1,8)}=5.45, p=0.047, \eta_{P}^{2}=\right.$ 0.12). Converting these results to percentage preference scores revealed no effect of $\mathrm{CNO}\left(t_{(9)}=0.57, p=0.293\right)$. Consistent with the lack of effect of TTX in the NAc on adult SAP, chemogenetic inhibition of the IC $\rightarrow$ NAc terminals appears to be insufficient to alter preference for the naive rat in adult SAP tests. Together, these results indicate that IC projections to NAc are necessary to show a preference to explore the stressed PN30 conspecific compared with the naive PN30.

\section{Chemogenetic activation of IC terminals in NAc increased exploration with PN30, but not PN50, conspecifics}

The results reviewed thus far suggest that during interactions with stressed juveniles IC projections to the NAc mediate the increase in social investigation directed toward the stressed targets. This set the stage to inquire whether this tract was sufficient to promote social exploration in the absence of social stress signals. We chose to again include PN50 conspecifics here because, whereas prior experiments suggested this pathway was not necessary for interactions with PN50 conspecifics, we did not have any prior data to rule out the possibility that the pathway was sufficient for interactions with PN50. To test this possibility, we augmented IC terminals in NAc by introducing the Gq coupled hM3Dq receptor via bilateral viral administration of pAAV5hSyn-hM3D(Gq)-mCherry in IC in 10 experimental rats. Cannula were placed bilaterally in NAc so that $\mathrm{CNO}$ could be directly applied to IC terminals in NAc as above (Fig. $5 A$ ). For each experimental rat included in the analysis, we confirmed the virus transduction via fluorescent imaging of the mCherry reporter (Fig. $5 A, C$, right), the location of the cannula tips in NAc (Fig. $5 B, C$ ), and the presence of terminal fibers expressing mCherry in NAc (Fig. $5 B$, inset). Three rats were excluded due to misplaced cannula or lack of virus expression. Three weeks after testing, experimental rats received social exploration tests over $5 \mathrm{~d}$ consisting of a habituation day, and then two tests with naive PN30 conspecifics and two tests with naive PN50 conspecifics. One trial for each conspecific age pairing occurred after vehicle treatment, and the other after infusion of $\mathrm{CNO}(0.5 \mu \mathrm{l} / \mathrm{side})$ to NAc $30 \mathrm{~min}$ before testing (Fig. 5G). The order of conspecific age and order of drug treatment were counterbalanced which allowed for a within-subjects design. Social exploration was analyzed as a twoway ANOVA with conspecific age and drug as within-subjects factors $\left(F_{(1,6)}=5.851, p=0.052, \eta_{p}^{2}=0.49\right)$. This analysis revealed main effects of conspecific age $\left(F_{(1,6)}=6.568, p=0.043\right.$, $\left.\eta_{P}^{2}=0.52\right)$ and $\operatorname{drug}\left(F_{(1,6)}=12.80, p=0.012, \eta_{P}^{2}=0.68\right)$. Post hoc comparisons show mean social exploration was greater with PN30 conspecifics after CNO treatment than vehicle $(p=0.030)$, whereas mean social exploration with PN50 conspecifics did not differ between $\mathrm{CNO}$ and vehicle treatment $(p=0.998)$ (Fig. $5 H$ ). To verify $\mathrm{hM} 3 \mathrm{D}(\mathrm{Gq})$ efficacy, a separate set of rats with $\mathrm{hM} 3 \mathrm{D}(\mathrm{Gq})$ expression in the IC were used for acute whole-cell patch-clamp recordings (Fig. $5 F, n=5$ cells) which confirmed 10 $\mu \mathrm{M}$ CNO reliably depolarized and inreased excitability of IC neurons visually identified as mCherry-positive at the time of recording (Fig. 5G). A one-way repeated-measures ANOVA revealed a main effect of $\mathrm{CNO}\left(F_{(2,8)}=4.33, p=0.053, h^{2}=0.52\right)$ and significant post hoc comparison between aCSF and CNO, $p=$ $0.032)$. Last, a day after testing ended, experimental rats received bilateral microinjections of vehicle $(n=4)$ or $\mathrm{CNO}(n=3) 2 \mathrm{~h}$ before being killed. NAc sections were stained for Fos to determine whether $\mathrm{CNO}$ was sufficient to increase activation of NAc neurons. CNO-treated rats expressed greater Fos-immunoreactivity compared with vehicle-treated rats $\left(t_{(5)}=2.727, p=0.041\right.$, $\eta^{2}=0.60$; Fig. $5 H$ ), supporting the efficacy of chemogenetic stimulation of IC-NAc terminals. These results indicate that excitation of IC terminals in NAc via $\mathrm{CNO}$ was sufficient to increase exploration with PN30, but not PN50 conspecifics.

\section{Insular cortex is not necessary for social novelty preference}

The foregoing results suggest the IC $\rightarrow$ NAc track is necessary and sufficient for social approach under conditions with a juvenile conspecific. Interestingly, manipulations of this tract did not influence behavior between two adult conspecifics. To establish whether IC is involved in situations of social reward per se, we used the social novelty preference test in which others have shown that an experimental rat will spend more time exploring an unfamiliar conspecific than a familiar animal and is dependent upon the NAc (Smith et al., 2018). We first adapted the social novelty preference test to be similar to the SAP test (Fig. 6A). Experimental adult rats were housed in groups of 4 for 2 weeks. Social novelty testing occurred over $3 \mathrm{~d}$; days 1 and 2 were habituation days exactly as in the SAP test. On day 3, each experimental adult rat was presented with a pair of same age conspecifics, one of which was a cage mate (familiar condition) and the other was from another cage (unfamiliar) and time spent interacting with each was quantified (Fig. $6 B)$. Experimental rats $(n=10)$ spent significantly more time exploring the unfamiliar conspecific $\left(t_{(9)}=3.703, p=0.005, \eta_{P}^{2}=0.60\right)$. To determine whether preference for novelty is mediated by the IC, a separate set of experimental rats received $\mathrm{hM} 4 \mathrm{D}(\mathrm{Gi})$ virus infusions to the IC exactly as in Figure 2. After recovery, rats were habituated to the arena and social interaction and then received either $\mathrm{CNO}$ ( $3 \mathrm{mg} / \mathrm{kg}$, i.p.) or vehicle injections $45 \mathrm{~min}$ before one of two social novelty preference tests (days 3 and 4, drug order counterbalanced; Fig. $6 C$ ). In this within-subjects design, every combination of familiar and unfamiliar conspecifics was unique such that the experimental rat was never tested with the same individuals. Three rats were removed from analysis because of undetect- 
A

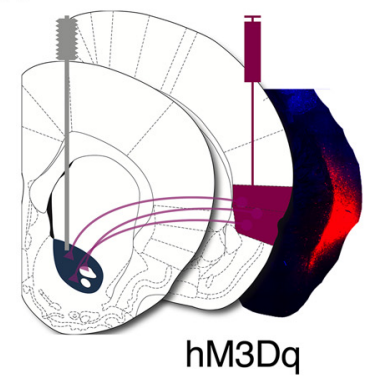

D

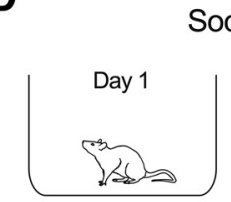

Arena Habituation
B

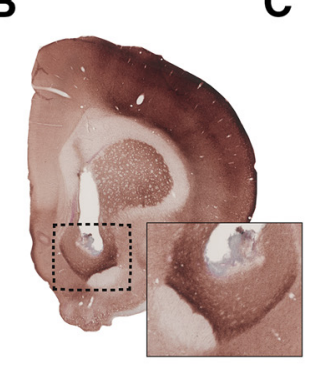

C

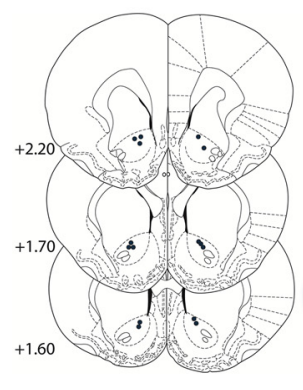

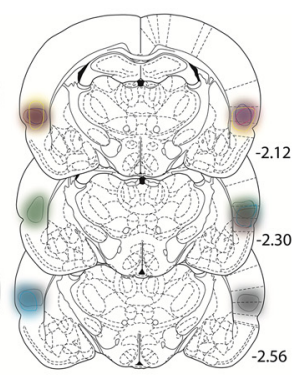

E

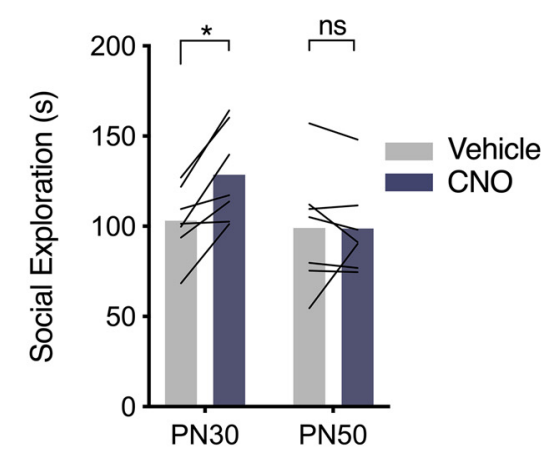

H

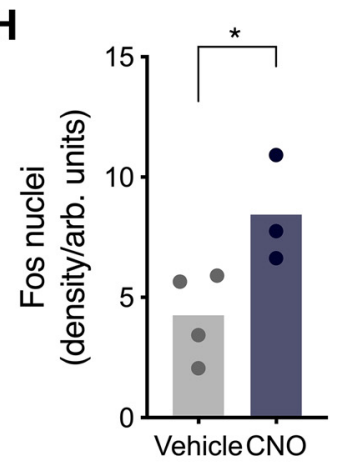

Figure 5. Chemogenetic excitation of IC terminals in NAc increased social exploration with PN30, but not PN50, conspecifics. A, Schematic of IC neurons transduced with pAAV5-hSyn-hM3D(Gq)$\mathrm{mCherry} \mathrm{in} \mathrm{IC} \mathrm{and} \mathrm{cannula} \mathrm{implanted} \mathrm{in} \mathrm{NAC} \mathrm{(left).} \mathrm{Terminal} \mathrm{excitation} \mathrm{of} \mathrm{mCherry-positive} \mathrm{fibers} \mathrm{was} \mathrm{achieved} \mathrm{via} \mathrm{bilateral} \mathrm{CNO} \mathrm{microinjections} \mathrm{to} \mathrm{NAc} \mathrm{(1} \mu \mathrm{m}$ ) 45 min before testing. Representative image of virus transduction (red is native mCherry expression, blue is DAPI) in IC (right). $\boldsymbol{B}$, Example of cannula tip placement in NAc and expression of terminal fibers in NAc (inset). $\boldsymbol{C}$, Map of cannula tip placements in NAc (left) and IC pAAV5-hSyn-hM3D(Gq)-mCherry expression in experimental rats (right). Each subject is represented by a different color ( $n=7$ ). $\boldsymbol{D}$, Diagram of social interaction trials. $\boldsymbol{E}$, Mean (with individual replicates) time spent exploring naive PN30 and PN50 conspecifics after vehicle and CN0 treatment (order counterbalanced). Experimental rats spent more time exploring naive PN30 conspecifics after CN0 injections than after vehicle treatment ( $p=0.030$ ), whereas social exploration with PN50 conspecifics was unaffected by drug treatment ( $p=0.998$ ). $\boldsymbol{F}$, Representative recording of CNO effect on $\mathrm{hM} 3 \mathrm{D}(\mathrm{Gq})$-expressing neuron in whole-cell recording. $\mathbf{G}$, After 10 min of bath application, CN0 increased the number of action potentials evoked by current injection in hM3Dq-mCherry-positive neurons in the IC. $(+)$ indicates the beginning of CNO perfusion. $\boldsymbol{H}$, Greater NAc Fos immunoreactivity was observed in rats injected with CNO before perfusion compared with rats treated with vehicle $(p=0.041)$. (ns) indicates non significant difference. ${ }^{*} p<0.05$.

able virus expression, or failure to show novelty preference under vehicle conditions; virus expression for the included rats is shown in Figure $6 D$. A repeated-measures ANOVA revealed a main effect of familiarity $\left(F_{(1,6)}=19.12, p=0.005, \eta_{p}^{2}=0.22\right)$; CNO had no effect on social novelty preference $(p=0.27)$.

\section{Discussion}

We investigated the role of the NAc and the projections from IC to NAc in social affective behavior. In an SAP test in which adult experimental rats demonstrate a preference to interact with stressed juvenile rats but avoid stressed adult rats (Rogers-Carter et al., 2018b), we observed effects of NAc and IC $\rightarrow$ NAc manipulations during interactions with juvenile conspecifics. First, pharmacological inactivation of the NAc prevented the expected preference for test rats to explore stressed PN30 conspecifics, but did not alter the avoidance of stressed PN50 conspecifics. Greater Fos immunoreactivity in NAc-projecting posterior IC neurons was evident in experimental rats after social interactions with stressed PN30 conspecifics compared with naive PN30 conspecifics. In SAP tests, chemogenetic inhibition of this circuit blocked the preference for the stressed PN30 conspecific but had no apparent effect on avoidance of stressed PN50 conspecifics. Accordingly, chemogenetic stimulation of IC terminals in NAc increased exploration with PN30, but not PN50, conspecifics. Interestingly, chemogenetic inhibition of the IC did not alter 
A

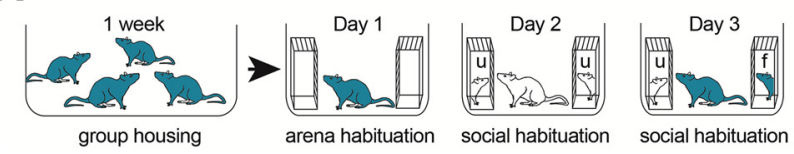

B

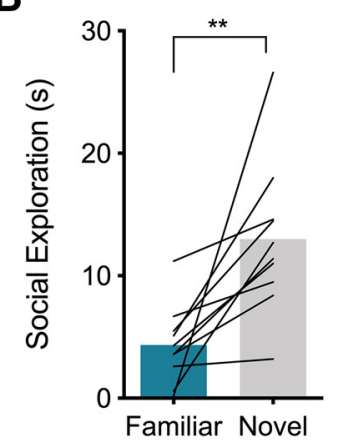

C

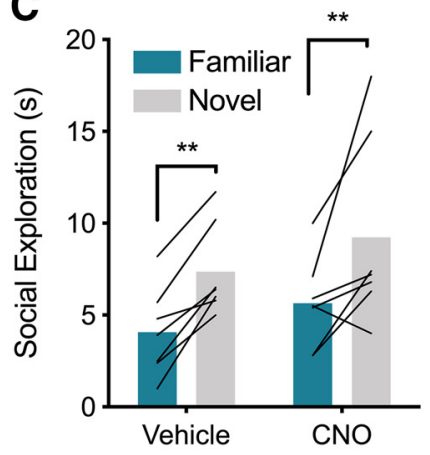

D

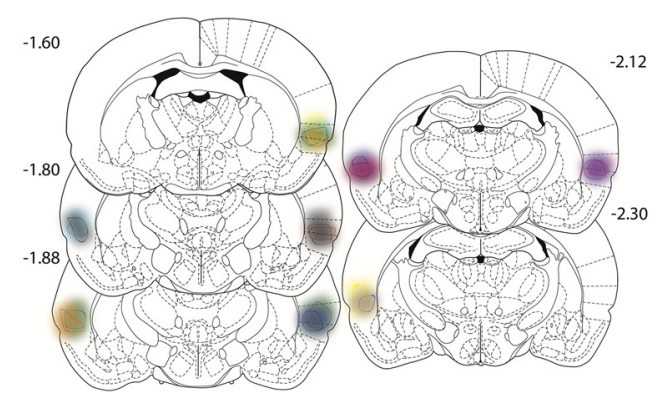

Figure 6. Insular cortex inhibition does not affect social novelty preference. $A$, Experimental overview. Rats were housed in groups to establish familiarity. After 2 weeks, experimental rats were habituated to the test arena and social interaction then given a 5 min novelty preference test with one of the experimental rat's cage mates (f, familiar) and an unfamiliar ( $u$ ) rat. $\boldsymbol{B}$, Mean (individual replicates) time spent in social interaction with the familiar and novel conspecifics. Experimental rats spent significantly more time investigating the unfamiliar target. ${ }^{* *} p=$ 0.005. C, To test the role of IC in social novelty preference, rats received pAAV5-hSyn-hM4D(Gi)$m$ Cherry virus injections to $\mathrm{IC}$ and then housed in groups for at least 2 weeks. Social novelty preference tests were given $45 \mathrm{~min}$ after pretreatment with either vehicle or $\mathrm{CNO}$ ( $3 \mathrm{mg} / \mathrm{kg}$, i.p.) on days 3 and 4. Experimental rats preferred interaction with the unfamiliar conspecific under both injection conditions ( ${ }^{* *}$ main effect of familiarity, $p=0.005$ ). $D$, Summary of mCherry expression indicating spread of $\mathrm{hM} 4 \mathrm{D}(\mathrm{Gi})$ in the $\mathrm{IC}$.

social approach to a novel conspecific. These results implicate the IC $\rightarrow$ NAc pathway in prosocial behaviors directed toward juveniles. In light of our existing understanding of social decisionmaking, we suggest that the IC serves an important role in integrating sensory information about social stimuli with nodes in subcortical decision-making networks, such as NAc, to modulate situationally appropriate social behaviors.

The involvement of NAc in social affective behavior is consistent with evidence implicating NAc in myriad social processes. NAc mediates social recognition (Ploeger et al., 1991), social reward (Dölen et al., 2013; Smith et al., 2018), mating and sexual behavior (Xiao and Becker, 1997; Fujiwara and Chiba, 2018), social novelty-seeking (Smith et al., 2017), and interactions with both juvenile (Trezza et al., 2011) and adult (Van den Berg et al., 1999; Dölen et al., 2013) conspecifics. Based on comparative neuroanatomy and genetic studies, O'Connell and Hofmann (2011) placed the NAc as a common node of the social decision-making network which integrates the dopaminergic reward circuitry with the neuroendocrine centers known to mediate social behavior. Importantly, NAc appears to decipher aversive from appetitive stimuli (Xiu et al., 2014) and modulate activity patterns in other social decision-making structures based on the valence of sensory information (Johnson et al., 2017). In the SAP paradigm, the preference to explore stressed juveniles may be mediated by the NAc because this region integrates the valence of conspecific affect with reward valuation (Hamel et al., 2017). Consistent with this thought, when NAc was pharmacologically inactivated with TTX, rats did not show a social preference for the stressed PN30 conspecific and thus behaved as if each conspecific was equal in valence.

Like NAc, IC output neurons are necessary for the social affective preference for the stressed juvenile (Rogers-Carter et al., 2018b). IC is a site of multisensory integration (Rodgers et al., 2008; Gogolla et al., 2014; Gogolla, 2017) and is reciprocally connected to the extended amygdala (Shi and Cassell, 1998), which may underlie its known role in human emotional recognition (Adolphs et al., 2003; Gu et al., 2013; Terasawa et al., 2015). Although the exact mechanism by which rodents convey emotion or affect is unknown, in the SAP test, we reported that there are changes to several overt conspecific behaviors (e.g., selfgrooming) and to the pattern of ultrasonic vocalizations, which together could convey important features of conspecific stress and age (Rogers-Carter et al., 2018b). There is now substantial evidence that IC encodes and distributes negative affective states to to subcortical networks to modulate behavior (Berret et al., 2019; Gehrlach et al., 2019). NAc is major target of IC efferents and, consistent with the hypothesis that IC projections to NAc mediate approach toward the stressed juvenile, we observed greater Fos IC $\rightarrow$ NAc neurons after interactions with stressed juveniles, with the greatest difference observed in the posterior IC. A limitation of the current experiment is small sample size; this experiment may have been underpowered to detect smaller effects of conspecific stress on IC $\rightarrow$ NAc neurons in the anterior IC, which tend to be greater after interactions with stressed juveniles. Future studies are needed to directly address whether these projections are involved in social approach behavior. Further, using both inhibitory and excitatory tract-specific chemogenetic manipulations of IC $\rightarrow$ NAc terminals, we find that this tract is both necessary for the preference for the stressed juvenile and sufficient to increase overall social exploration directed toward juvenile conspecifics. Together, these findings demonstrate the modulatory role of IC $\rightarrow$ NAc projections on social approach in a preference paradigm.

Rats require parental support to develop and survive (Lamers et al., 1986), and the same mechanisms that evolved to enable parental care may also underlie more complex social abilities (Scheiber et al., 2017; Marsh, 2019). Because these social actions are necessary for species survival, they are likely reinforced by the reward system to promote their occurrence (O'Connell and Hofmann, 2011). Although we cannot be sure that approach to stressed juveniles is a prosocial response, increased NAc activity is associated with many prosocial behaviors. Optogenetic excitation of ventral tegmental neurons that project to the nucleus accumbens promoted mating behavior (Beny-Shefer et al., 2017) and microinfusion of a dopamine reuptake inhibitor increased social play in rats (Manduca et al., 2016). Similarly, oxytocin in NAc increases social approach (Steinman et al., 2019) and is important for forming pair bonds (Insel and Shapiro, 1992; Walum et al., 2012; Walum and Young, 2018). Our findings that chemogenetic manipulation of IC terminals in NAc relates to adult-tojuvenile behaviors adds to these NAc-dependent prosocial behaviors and suggest an anatomical tract for socioemotional information to be integrated in social decision-making. 
Although the primary goal of this study was to investigate neural substrates of social behavior, this work adds to several studies which seek to identify functions of the IC using intersectional behavioral neuroscience methods. Recent reports in drug administration models show excitation of IC projections to central amygdala promote relapse of methamphetamine use (Venniro et al., 2017) and IC projections to the bed nucleus of the stria terminalis mediate negative affect during alcohol abstinence (Centanni et al., 2019). Similarly, silencing of the IC projection to NAc decreased alcohol self-administration (Jaramillo et al., 2018b) and increased sensitivity to alcohol (Jaramillo et al., 2018a), and IC projections to the central amygdala drive avoidance of an aversive tastant (Schiff et al., 2018) and fear (Berret et al., 2019; Gehrlach et al., 2019). Interestingly, in an appetitive consummatory task (sucrose preference) optogenetic stimulation of IC to NAc projections reduced consumption (Gehrlach et al., 2019). Although more work will add clarification, an emergent theme is that IC mediates both appetitive and aversive behavioral responses to external stimuli via different projections. IC contributes to both social approach and avoidance (RogersCarter et al., 2018b), the latter of which may be mediated IC projections to the central amygdala, which is necessary for social avoidance of stressed conspecifics (Ferretti et al., 2019).

An interesting issue in the SAP test concerns the motivation of experimental rats to approach stressed juveniles. Indeed, in much of the social behavior literature social stress signals lead to avoidance. We speculated that adult-generated social affective cues were perceived as danger signals, whereas juvenile stress signals elicited stress buffering or parental behaviors from adults (Rogers-Carter et al., 2018a,b). These results parallel several features of human social behavior, namely that social stress cues modulate rodent social interactions in ways that mirror adult preference to lend help to juveniles while showing less empathy toward other adults (Batson and Powell, 2003). A close observation of social preference data reveals that, although the majority of rats approach stressed juveniles and avoid stressed adults, some individuals are unaffected by conspecific stress, or exhibit the opposite preference compared with the majority. Previously, we found SAP behavior to be normally distributed and speculated that individual variability was a consequence of biological variables, such as oxytocin receptor levels in the insula, that vary normally in rats (Rogers-Carter et al., 2018b). Identifying the biological basis for the behavior in the individuals at the tails of the distribution may relate to pathophysiology in socioemotional behavior. Abnormalities in social processes such as emotion recognition are symptoms of several psychiatric conditions including autism spectrum disorders (Tracy et al., 2011; Lozier et al., 2014; Griffiths et al., 2019; Sato et al., 2017), and neuroimaging studies of individuals with autism find reduced insular activation to emotional stimuli (Di Martino et al., 2009; Morita et al., 2012; Hall et al., 2013), reduced striatal activation to social rewards (Schmitz et al., 2008; Scott-Van Zeeland et al., 2010; Supekar et al., 2018), and reduced functional connectivity between insula and ventral striatum (Fuccillo, 2016). Our results provide mechanistic support for this axis as a key to normative social cognition. Future research might take advantage of the SAP phenomenon to identify the tracts that mediate the avoidance of stressed adults and determine how genetic and experimental risk factors for autism spectrum disorders influence the IC and its inputs and outputs.

\section{References}

Adolphs R, Tranel D, Damasio AR (2003) Dissociable neural systems for recognizing emotions. Brain Cogn 52:61-69.

Batson CD, Powell AA (2003) Altruism and prosocial behavior. In: Handbook of psychology volume 5: personality and social psychology. (Millon T, Lerner MJ, Weiner IB, eds), pp 463-484. Hoboken, NJ: John Wiley \& Sons, Inc.

Beny-Shefer Y, Zilkha N, Lavi-Avnon Y, Bezalel N, Rogachev I, Brandis A, Dayan M, Kimchi T (2017) Nucleus accumbens dopamine signaling regulates sexual preference for females in male mice. Cell Rep 21: 3079-3088.

Berret E, Kintscher M, Palchaudhuri S, Tang W, Osypenko D, Kochubey O, Schneggenburger R (2019) Insular cortex processes aversive somatosensory information and is crucial for threat learning. Science 364:eaaw0474.

Burkett JP, Andari E, Johnson ZV, Curry DC, de Waal FB, Young LJ (2016) Oxytocin-dependent consolation behavior in rodents. Science 351: 375-378.

Centanni SW, Morris BD, Luchsinger JR, Bedse G, Fetterly TL, Patel S, Winder DG (2019) Endocannabinoid control of the insular-bed nucleus of the stria terminalis circuit regulates negative affective behavior associated with alcohol abstinence. Neuropsychopharmacology 44:526537.

Christianson JP, Jennings JH, Ragole T, Flyer JG, Benison AM, Barth DS, Watkins LR, Maier SF (2011) Safety signals mitigate the consequences of uncontrollable stress via a circuit involving the sensory insular cortex and bed nucleus of the stria terminalis. Biol Psychiatry 70:458-464.

Di Martino A, Shehzad Z, Kelly C, Roy AK, Gee DG, Uddin LQ, Gotimer K, Klein DF, Castellanos FX, Milham MP (2009) Relationship between cingulo-insular functional connectivity and autistic traits in neurotypical adults. Am J Psychiatry 166:891-899.

Dölen G, Darvishzadeh A, Huang KW, Malenka RC (2013) Social reward requires coordinated activity of nucleus accumbens oxytocin and serotonin. Nature 501:179-184.

Dong X, Li S, Kirouac GJ (2017) Collateralization of projections from the paraventricular nucleus of the thalamus to the nucleus accumbens, bed nucleus of the stria terminalis, and central nucleus of the amygdala. Brain Struct Funct 222:3927-3943.

Felix-Ortiz AC, Burgos-Robles A, Bhagat ND, Leppla CA, Tye KM (2016) Bidirectional modulation of anxiety-related and social behaviors by amygdala projections to the medial prefrontal cortex. Neuroscience 321: 197-209.

Ferretti V, Maltese F, Contarini G, Nigro M, Bonavia A, Huang H, Gigliucci V, Morelli G, Scheggia D, Managò F, Castellani G, Lefevre A, Cancedda L, Chini B, Grinevich V, Papaleo F (2019) Oxytocin signaling in the central amygdala modulates emotion discrimination in mice. Curr Biol 29:19381953.e6.

Fuccillo MV (2016) Striatal circuits as a common node for autism pathophysiology. Front Neurosci 10:27.

Fujiwara M, Chiba A (2018) Sexual odor preference and dopamine release in the nucleus accumbens by estrous olfactory cues in sexually naïve and experienced male rats. Physiol Behav 185:95-102.

Gehrlach DA, Dolensek N, Klein AS, Roy Chowdhury R, Matthys A, Junghänel M, Gaitanos TN, Podgornik A, Black TD, Reddy Vaka N, Conzelmann KK, Gogolla N (2019) Aversive state processing in the posterior insular cortex. Nat Neurosci 22:1424-1437.

Gogolla N (2017) The insular cortex. Curr Biol 27:R580-R586.

Gogolla N, Takesian AE, Feng G, Fagiolini M, Hensch TK (2014) Sensory integration in mouse insular cortex reflects GABA circuit maturation. Neuron 83:894-905.

Griffiths S, Jarrold C, Penton-Voak IS, Woods AT, Skinner AL, Munafò MR (2019) Impaired recognition of basic emotions from facial expressions in young people with autism spectrum disorder: assessing the importance of expression intensity. J Autism Dev Disord 49:2768-2778.

Gu X, Hof PR, Friston KJ, Fan J (2013) Anterior insular cortex and emotional awareness. J Comp Neurol 521:3371-3388.

Guzmán YF, Tronson NC, Guedea A, Huh KH, Gao C, Radulovic J (2009) Social modeling of conditioned fear in mice by non-fearful conspecifics. Behav Brain Res 201:173-178.

Hall SS, Jiang H, Reiss AL, Greicius MD (2013) Identifying large-scale brain networks in fragile X syndrome. JAMA Psychiatry 70:1215-1223.

Hamel L, Thangarasa T, Samadi O, Ito R (2017) Caudal nucleus accumbens 
core is critical in the regulation of cue-elicited approach-avoidance decisions. eNeuro 4:ENEURO.0330-16.2017.

Hong W, Kim DW, Anderson DJ (2014) Antagonistic control of social versus repetitive self-grooming behaviors by separable amygdala neuronal subsets. Cell 158:1348-1361.

Insel TR, Fernald RD (2004) How the brain processes social information: searching for the social brain. Annu Rev Neurosci 27:697-722.

Insel TR, Shapiro LE (1992) Oxytocin receptor distribution reflects social organization in monogamous and polygamous voles. Proc Natl Acad Sci U S A 89:5981-5985.

Ishii H, Ohara S, Tobler PN, Tsutsui K, Iijima T (2012) Inactivating anterior insular cortex reduces risk taking. J Neurosci 32:16031-16039.

Jaramillo AA, Randall PA, Stewart S, Fortino B, Van Voorhies K, Besheer J (2018a) Functional role for cortical-striatal circuitry in modulating alcohol self-administration. Neuropharmacology 130:42-53.

Jaramillo AA, Van Voorhies K, Randall PA, Besheer J (2018b) Silencing the insular-striatal circuit decreases alcohol self-administration and increases sensitivity to alcohol. Behav Brain Res 348:74-81.

Johnson ZV, Walum H, Xiao Y, Riefkohl PC, Young LJ (2017) Oxytocin receptors modulate a social salience neural network in male prairie voles. Horm Behav 87:16-24.

Kiyokawa Y, Honda A, Takeuchi Y, Mori Y (2014) A familiar conspecific is more effective than an unfamiliar conspecific for social buffering of conditioned fear responses in male rats. Behav Brain Res 267:189-193.

Kohls G, Chevallier C, Troiani V, Schultz RT (2012) Social 'wanting' dysfunction in autism: neurobiological underpinnings and treatment implications. J Neurodev Disord 4:10.

Lamers WH, Mooren PG, Griep H, Endert E, Degenhart HJ, Charles R (1986) Hormones in perinatal rat and spiny mouse: relation to altricial and precocial timing of birth. Am J Physiol 251:E78-E85.

Langford DJ, Crager SE, Shehzad Z, Smith SB, Sotocinal SG, Levenstadt JS, Chanda ML, Levitin DJ, Mogil JS (2006) Social modulation of pain as evidence for empathy in mice. Science 312:1967-1970.

Lee H, Kim DW, Remedios R, Anthony TE, Chang A, Madisen L, Zeng H, Anderson DJ (2014) Scalable control of mounting and attack by Esr1+ neurons in the ventromedial hypothalamus. Nature 509:627-632.

Li Z, Lu YF, Li CL, Wang Y, Sun W, He T, Chen XF, Wang XL, Chen J (2014) Social interaction with a cagemate in pain facilitates subsequent spinal nociception via activation of the medial prefrontal cortex in rats. Pain 155:1253-1261.

Lozier LM, Vanmeter JW, Marsh AA (2014) Impairments in facial affect recognition associated with autism spectrum disorders: a meta-analysis. Dev Psychopathol 26:933-945.

MacLaren DA, Browne RW, Shaw JK, Krishnan Radhakrishnan S, Khare P, España RA, Clark SD (2016) Clozapine N-oxide administration produces behavioral effects in Long-Evans rats: implications for designing DREADD experiments. eNeuro 3:ENEURO.0219-16.2016.

Mahler SV, Aston-Jones G (2018) CNO evil? considerations for the use of DREADDs in behavioral neuroscience. Neuropsychopharmacology 43: 934-936.

Mahler SV, Vazey EM, Beckley JT, Keistler CR, McGlinchey EM, Kaufling J, Wilson SP, Deisseroth K, Woodward JJ, Aston-Jones G (2014) Designer receptors show role for ventral pallidum input to ventral tegmental area in cocaine seeking. Nat Neurosci 17:577-585.

Manduca A, Servadio M, Damsteegt R, Campolongo P, Vanderschuren LJ, Trezza V (2016) Dopaminergic neurotransmission in the nucleus accumbens modulates social play behavior in rats. Neuropsychopharmacology 41:2215-2223.

Manvich DF, Webster KA, Foster SL, Farrell MS, Ritchie JC, Porter JH, Weinshenker D (2018) The DREADD agonist clozapine N-oxide (CNO) is reverse-metabolized to clozapine and produces clozapine-like interoceptive stimulus effects in rats and mice. Sci Rep 8:3840.

Marsh AA (2019) The caring continuum: evolved hormonal and proximal mechanisms explain prosocial and antisocial extremes. Annu Rev Psychol 70:347-371.

Meyza KZ, Bartal IB, Monfils MH, Panksepp JB, Knapska E (2017) The roots of empathy: Through the lens of rodent models. Neurosci Biobehav Rev 76:216-234.

Morita T, Kosaka H, Saito DN, Ishitobi M, Munesue T, Itakura S, Omori M, Okazawa H, Wada Y, Sadato N (2012) Emotional responses associated with self-face processing in individuals with autism spectrum disorders: an fMRI study. Soc Neurosci 7:223-239.
O'Connell LA, Hofmann HA (2011) The vertebrate mesolimbic reward system and social behavior network: a comparative synthesis. J Comp Neurol 519:3599-3639.

O'Connell LA, Hofmann HA (2012) Evolution of a vertebrate social decision-making network. Science 336:1154-1157.

Park JY, Park SY, Kwon H, Song Y, Yun B, Lee Y, Cho Y, Joo A, Han PL (2018) A group of descending glutamatergic neurons activated by stress in corticolimbic regions project to the nucleus accumbens. Exp Neurobiol 27:387-396.

Parks SL, Bradfield LA, Balleine BW (2015) Interaction of insular cortex and ventral striatum mediates the effect of incentive memory on choice between goal-directed actions. J Neurosci 35:6464-6471.

Ploeger GE, Willemen AP, Cools AR (1991) Role of the nucleus accumbens in social memory in rats. Brain Res Bull 26:23-27.

Rodgers KM, Benison AM, Klein A, Barth DS (2008) Auditory, somatosensory, and multisensory insular cortex in the rat. Cereb Cortex 18: 2941-2951.

Rogers-Carter MM, Djerdjaj A, Culp AR, Elbaz JA, Christianson JP (2018a) Familiarity modulates social approach toward stressed conspecifics in female rats. PLoS One 13:e0200971.

Rogers-Carter MM, Varela JA, Gribbons KB, Pierce AF, McGoey MT, Ritchey M, Christianson JP (2018b) Insular cortex mediates approach and avoidance responses to social affective stimuli. Nat Neurosci 21:404-414.

Rogers-Carter MM, Christianson JP (2019) An insular view of the social decision-making network. Neurosci Biobehav Rev 103:119-132.

Roth BL (2016) DREADDs for neuroscientists. Neuron 89:683-694.

Sato W, Sawada R, Uono S, Yoshimura S, Kochiyama T, Kubota Y, Sakihama M, Toichi M (2017) Impaired detection of happy facial expressions in autism. Sci Rep 7:13340.

Scheiber IBR, Weiß BM, Kingma SA, Komdeur J (2017) The importance of the altricial - precocial spectrum for social complexity in mammals and birds - a review. Front Zool 14:3.

Schiff HC, Bouhuis AL, Yu K, Penzo MA, Li H, He M, Li B (2018) An insula-central amygdala circuit for guiding tastant-reinforced choice behavior. J Neurosci 38:1418-1429.

Schmitz N, Rubia K, van Amelsvoort T, Daly E, Smith A, Murphy DG (2008) Neural correlates of reward in autism. Br J Psychiatry 192:19-24.

Scott-Van Zeeland AA, Dapretto M, Ghahremani DG, Poldrack RA, Bookheimer SY (2010) Reward processing in autism. Autism Res 3:5367.

Shi CJ, Cassell MD (1998) Cortical, thalamic, and amygdaloid connections of the anterior and posterior insular cortices. J Comp Neurol 399:440 468.

Smith CJW, Mogavero JN, Tulimieri MT, Veenema AH (2017) Involvement of the oxytocin system in the nucleus accumbens in the regulation of juvenile social novelty-seeking behavior. Horm Behav 93:94-98.

Smith CJW, Wilkins KB, Li S, Tulimieri MT, Veenema AH (2018) Nucleus accumbens mu opioid receptors regulate context-specific social preferences in the juvenile rat. Psychoneuroendocrinology 89:59-68.

Smith KS, Bucci DJ, Luikart BW, Mahler SV (2016) DREADDS: use and application in behavioral neuroscience. Behav Neurosci 130:137-155.

Stachniak TJ, Ghosh A, Sternson SM (2014) Chemogenetic synaptic silencing of neural circuits localizes a hypothalamus $\rightarrow$ midbrain pathway for feeding behavior. Neuron 82:797-808.

Steinman MQ, Duque-Wilckens N, Trainor BC (2019) Complementary neural circuits for divergent effects of oxytocin: social approach versus social anxiety. Biol Psychiatry 85:792-801.

Supekar K, Kochalka J, Schaer M, Wakeman H, Qin S, Padmanabhan A, Menon V (2018) Deficits in mesolimbic reward pathway underlie social interaction impairments in children with autism. Brain 141:2795-2805.

Terasawa Y, Kurosaki Y, Ibata Y, Moriguchi Y, Umeda S (2015) Attenuated sensitivity to the emotions of others by insular lesion. Front Psychol 6:1314.

Tracy JL, Robins RW, Schriber RA, Solomon M (2011) Is emotion recognition impaired in individuals with autism spectrum disorders. J Autism Dev Disord 41:102-109.

Trezza V, Damsteegt R, Achterberg EJ, Vanderschuren LJ (2011) Nucleus accumbens $\mu$-opioid receptors mediate social reward. J Neurosci 31: 6362-6370.

Van den Berg CL, Pijlman FT, Koning HA, Diergaarde L, Van Ree JM, Spruijt BM (1999) Isolation changes the incentive value of sucrose and social behaviour in juvenile and adult rats. Behav Brain Res 106:133-142. 
Venniro M, Caprioli D, Zhang M, Whitaker LR, Zhang S, Warren BL, Cifani C, Marchant NJ, Yizhar O, Bossert JM, Chiamulera C, Morales M, Shaham Y (2017) The anterior insular cortex $\longrightarrow>$ central amygdala glutamatergic pathway is critical to relpase after contingency management. Neuron 96:414-427.e8.

Walum H, Young LJ (2018) The neural mechanisms and circuitry of the pair bond. Nat Rev Neurosci 19:643-654.

Walum H, Lichtenstein P, Neiderhiser JM, Reiss D, Ganiban JM, Spotts EL, Pedersen NL, Anckarsäter H, Larsson H, Westberg L (2012) Variation in the oxytocin receptor gene is associated with pair-bonding and social behavior. Biol Psychiatry 71:419-426.

Willuhn I, Tose A, Wanat MJ, Hart AS, Hollon NG, Phillips PE, Schwarting RK, Wöhr M (2014) Phasic dopamine release in the nucleus accumbens in response to pro-social $50 \mathrm{kHz}$ ultrasonic vocalizations in rats. J Neurosci 34:10616-10623.

Wise RA (2002) Brain reward circuitry: insights from unsensed incentives. Neuron 36:229-240.

Wright CI, Groenewegen HJ (1996) Patterns of overlap and segregation be- tween insular cortical, intermediodorsal thalamic and basal amygdaloid afferents in the nucleus accumbens of the rat. Neuroscience 73:359 373

Wu YL, Yoshida M, Emoto H, Tanaka M (1999) Psychological stress selectively increases extracellular dopamine in the 'shell', but not in the 'core' of the rat nucleus accumbens: a novel dual-needle probe simultaneous microdialysis study. Neurosci Lett 275:69-72.

Xiao L, Becker JB (1997) Hormonal activation of the striatum and the nucleus accumbens modulates paced mating behavior in the female rat. Horm Behav 32:114-124.

Xiu J, Zhang Q, Zhou T, Zhou TT, Chen Y, Hu H (2014) Visualizing an emotional valence map in the limbic forebrain by TAI-FISH. Nat Neurosci 17:1552-1559.

Yang AC, Tsai SJ (2017) New targets for schizophrenia treatment beyond the dopamine hypothesis. Int J Mol Sci 18:E1689.

Yao S, Bergan J, Lanjuin A, Dulac C (2017) Oxytocin signaling in the medial amygdala is required for sex discrimination of social cues. Elife 6:e31373. 